\title{
Decomposition of homogeneous vector fields of degree one and representation of the flow ${ }^{*}$
}

by

\author{
Fabio ANCONA \\ Department of Mathematics, P.O. Box 395, University of Colorado, \\ Boulder, Colorado 80309-0395, ancona@euclid.colorado.edu \\ and \\ S.I.S.S.A. - International School for Advanced Studies, \\ via Beirut n. 2-4, Trieste 34014, Italy, \\ ancona@neumann.sissa.it
}

ABSTRACT. - We first give a characterization for the set of real analytic diffeomorphisms which transform homogeneous vector fields of certain degree into homogeneous fields of the same degree with respect to an arbitrary dilation $\delta_{\varepsilon}^{r}$. Such a set is constituted by the invertible analytic maps that are homogeneous of degree one with respect to $\delta_{\varepsilon}^{r}$ and can be endowed with the structure of a Lie Group whose Lie algebra is the space $H^{1, r}\left(\mathbb{R}^{n}\right)$ of the homogeneous fields of degree one with respect to $\delta_{\varepsilon}^{r}$. Then we prove a decomposition theorem for the elements of the non semisimple Lie algebra $H^{1, r}\left(\mathbb{R}^{n}\right)$. This result is a non linear analog of the Jordan decomposition of a linear field, i.e. for $X \in H^{1, r}\left(\mathbb{R}^{n}\right)$, we can write $X=S+N$, with $S$ linear semisimple and $[S, N]=0$. We also give an explicit representation formula for the flow generated by a field in $H^{1, r}\left(\mathbb{R}^{n}\right)$. Finally we apply this result to obtain a simple representation for the trajectories of a class of affine control systems $\dot{x}=X_{0}(x)+B u$, with $X_{0} \in H^{1, r}\left(\mathbb{R}^{n}\right)$ and $B$ a constant field, that constitute a natural extension of the linear control systems.

Key words: Homogeneous vector fields, decomposition of vector fields, nonlinear systems, representation of solutions, Lie algebra of a Lie group.

* This research was partially supported by National Science Foundation Grant DMS 9301039.

1991 Mathematics Subject Classification: 34 A 05, 34 C 20, 47 A 20, 57 R 25.

Annales de l'Institut Henri Poincaré - Analyse non linéaire - 0294-1449 
RÉsuMÉ. - Tout d'abord, nous donnons une caractérisation de l'ensemble des difféomorphismes analytiques réels qui transforment des champs de vecteurs homogènes d'un certain degré en champs de vecteurs homogènes de même degré relativement à une dilatation arbitraire $\delta_{\varepsilon}^{r}$. Un tel ensemble est constitué par des applications analytiques inversibles homogènes de degré 1 relativement à $\delta_{\varepsilon}^{r}$, et il peut être doté d'une structure de groupe de Lie. L'espace $H^{1, r}\left(\mathbb{R}^{n}\right)$ des champs de vecteurs homogènes de degré 1 relativement à $\delta_{\varepsilon}^{r}$ est l'algèbre de Lie de cet ensemble. Ensuite, nous démontrons un théorème de décomposition pour les éléments de l'algèbre de Lie non-semisimple $H^{1, r}\left(\mathbb{B}^{n}\right)$. Ce résultat est l'analogue non linéaire de la décomposition de Jordan d'un champ linéaire, i.e., pour $X \in H^{1, r}\left(\mathbb{R}^{n}\right)$, nous pouvons écrire $X=S+N$, où $S$ est un champ linéaire semisimple et $[S, N]=0$. Nous donnons aussi une formule explicite de représentation pour le flux d'un champ de $H^{1, r}\left(\mathbb{R}^{n}\right)$. Finalement, nous utilisons ce résultat pour obtenir une représentation simple des trajectoires d'une classe du système affines de contrôle $\dot{x}=X_{0}(x)+B u$, où $X_{0} \in H^{1, r}\left(\mathbb{R}^{n}\right)$ et $B$ est un champ constant, qui constitue une extension naturelle des systèmes linéaires de contrôle.

\section{INTRODUCTION}

Consider an affine nonlinear control system

$$
\dot{x}=X_{0}(x)+\sum_{j=1}^{m} u_{j} X_{j}(x),
$$

where $X_{0}, X_{1}, \ldots X_{m}$ are real analytic vector fields on $\mathbb{R}^{n}$ and $u=$ $\left(u_{1}, \ldots, u_{m}\right)$ is the control. A well known technique for the local study of such a system consists in locally approximating the vector fields $X_{j}, j=0,1, \ldots, m$, by fields $Y_{j}, j=0,1, \ldots, m$, for which the analysis is easier and such that they "preserve" the property being studied. This technique has been the key in obtaining high order local controllability results and in the construction of asymptotically stabilizing feedback controls, e.g., see [5], [19], [10], [11], [12], [13], [16]. Homogeneous vector fields with respect to a dilation $\delta_{\varepsilon}^{r}$ have often provided such "correct" approximations in basically non linear problems for which the usual linear approximations fail to yield sufficient information. Results in this direction can be found in [4], [9], [20]. 
In this paper we study some basic properties of the homogeneous vector fields of degree one with respect to an arbitrary dilation $\delta_{\varepsilon}^{r}$ and develop a general method of solution for every autonomous system of differential equations

$$
\dot{x}=X(x),
$$

where $X$ is a real analytic vector field on $\mathbb{R}^{n}$ (or on an $n$-dimensional manifold $M^{n}$ ) homogeneous of degree one with respect to $\delta_{\varepsilon}^{r}$.

Specifically, let $\delta_{\varepsilon}^{r}: \mathbb{R}^{n} \rightarrow \mathbb{R}^{n}$ be a dilation on $\mathbb{R}^{n}$ defined by $\delta_{\varepsilon}^{r}(x)=\left(\varepsilon^{r_{1}} x_{1}, \ldots, \varepsilon^{r_{n}} x_{n}\right)$, where $\varepsilon>0$, and $r_{1} \leq \ldots \leq r_{n}$ are positive integers. A polynomial $p: \mathbb{R}^{n} \rightarrow \mathbb{R}$ is homogeneous of degree $m \in \mathbb{N}$ with respect to $\delta_{\varepsilon}^{r}$ if $p\left(\delta_{\varepsilon}^{r}(x)\right)=\varepsilon^{m} p(x)$. (Throughout $\mathbb{N}$ will denote the set of non negative integers $\{0,1, \ldots\}$.) The set of polynomials homogeneous of degree $m$ with respect to $\delta_{\varepsilon}^{r}$ will be denoted $P^{m, r}\left(\mathbb{R}^{n}\right)$. We define a real analytic vector field $X(x)=\sum_{i=1}^{n} a_{i}(x) \partial / \partial x_{i}$ on $\mathbb{R}^{n}$, given in local coordinates $x=\left(x_{1}, \ldots x_{n}\right)$, to be homogeneous of degree $m \in \mathbb{Z}$ with respect to $\delta_{\varepsilon}^{r}$ if $a_{i} \in P^{r_{i}+m-1, r}\left(\mathbb{R}^{n}\right), i=1, \ldots, n$. We denote by $H^{m, r}\left(\mathbb{R}^{n}\right)$ the family of such vector fields. This definition (although not universally used) agrees with the classical definition of homogeneity (i.e., $\left.a_{i}(\varepsilon x)=\varepsilon^{m} a_{i}(x), i=1, \ldots, n\right)$ in the case $X$ is homogeneous with respect to the standard dilation $\delta_{\varepsilon}^{1}$, having $r_{1}=\ldots=r_{n}=1$. In particular, a field $X(x)=A x$ that is linear in the local coordinates will be homogeneous of degree one w.r.t. $\delta_{\varepsilon}^{1}$. One can thus regard the concept of homogeneity of degree one w.r.t. an arbitrary dilation as a natural extension of the concept of linearity. In fact, classical results valid for linear vector fields have been obtained for such fields in nonlinear problems, where the homogeneous fields play the role of the linear approximations in classical theory, e.g., see [10].

The paper is organized as follows: in Section 2 we characterize the set of all real analytic diffeomorphisms $\phi$ on $\mathbb{R}^{n}$ that transform any homogeneous vector field $X$ of degree $m$ with respect to a given dilation $\delta_{\varepsilon}^{r}$ into a homogeneous field $T_{\phi} X$ of the same degree with respect to the same dilation. If $\delta_{\varepsilon}^{r}$ is the standard dilation $\delta_{\varepsilon}^{1}$, such a set is clearly the set of all invertible linear transformations on $\mathbb{R}^{n}$, which in our notation coincides with the set of all invertible elements of $H^{1,1}\left(\mathbb{R}^{n}\right)$. We show that also in the case of an arbitrary dilation $\delta_{\varepsilon}^{r}, r=\left(r_{1}, \ldots, r_{n}\right)$, with $r_{1}=1$, the set of all real analytic changes of coordinates that transform homogeneous vector fields of certain degree into homogeneous fields of the same degree is precisely the set of all invertible elements of $H^{1, r}\left(\mathbb{R}^{n}\right)$, which will be denoted by $G H^{1, r}\left(\mathbb{R}^{n}\right)$. This set is a subgroup of the group of all real analytic diffeomorphisms on $\mathbb{R}^{n}$ and can be endowed Vol. 13, n²-1996. 
with the structure of a finite dimensional Lie group. Moreover, if $X$ is a field in $H^{1, r}\left(\mathbb{R}^{n}\right)$ and $(\exp t X)(p)$ denotes the solution, at time $t$, of the Cauchy problem $\dot{x}=X(x), x(0)=p$, then, for $t$ fixed, the diffeomorphism $p \rightarrow(\exp t X)(p)$ lies in $G H^{1, r}\left(\mathbb{R}^{n}\right)$. It follows as an easy consequence that the space $H^{1, r}\left(\mathbb{R}^{n}\right)$ of the homogeneous fields of degree one with respect to a dilation $\delta_{\varepsilon}^{r}$ is the Lie algebra of the Lie group $G H^{1, r}\left(\mathbb{R}^{n}\right)$.

In Section 3 we prove a decomposition theorem for the vector fields of the non semisimple Lie algebra $H^{1, r}\left(\mathbb{R}^{n}\right)$, providing a non linear analog to the Jordan decomposition of a linear vector field into a semisimple and nilpotent part.

THEOREM 3.1. - Let $X(x)=\sum_{i=1}^{n} a_{i}(x) \partial / \partial x_{i}$ be a real analytic vector field on $\mathbb{R}^{n}$ homogeneous of degree one with respect to a given dilation $\delta_{\varepsilon}^{r}$. Then there is a polynomial change of coordinates $x=\phi(y), \phi \in$ $G H^{1, r}\left(\mathbb{R}^{n}\right)$, such that

$$
T_{\phi} X=S+N
$$

$\left(T_{\phi} X(y)\right.$ denoting the transformed field after performing the coordinate change $x=\phi(y)$ ) where $S$ and $N$ are real analytic homogeneous vector fields of degree one with respect to $\delta_{\varepsilon}^{r}$, satisfying

$$
[S, N]=0 \text {. }
$$

Moreover $S$ is a linear semisimple vector field (i.e. the complexification of $S$ is diagonalizable) and $N$ is the sum of a linear nilpotent field and of a strictly non linear homogeneous vector field of degree one with respect to $\delta_{\varepsilon}^{r}$.

Finally, in Section 4 we obtain a simple representation of the solutions of system (1.2), in terms of their Picard approximations, for a class of fields that can be regarded as a generalization of the linear nilpotent vector fields. This result, together with the decomposition theorem given in Section 3, yields a representation formula for the solutions of (1.2), for any $X \in H^{1, r}\left(\mathbb{R}^{n}\right)$. We also derive an explicit representation for the trajectories $x(\cdot, u)$ of an $n$-dimensional, single input, affine control system

$$
\dot{x}=X_{0}(x)+B u
$$

where $X_{0}$ is an element of $H^{1, r}\left(\mathbb{R}^{n}\right), r=\left(r_{1}, \ldots, r_{n}\right)$, and $B$ is a constant field whose local coordinate expression is given by an $n \times 1$ matrix having nonzero entries $b_{i}$ only for those $i$ such that $r_{i}=r_{n}$ :

$$
x(t, u)=\left(\exp t X_{0}\right)\left(x(0)+\int_{0}^{t} e^{-s A} B u(s) d s\right), \quad t \in \mathbb{R},
$$

denoting by $A$ the linear part of the field $X_{0}$. 


\section{HOMOGENEOUS DIFFEOMORPHISMS OF DEGREE ONE WITH RESPECT TO AN ARBITRARY DILATION}

\subsection{Notations and Definitions}

The general setting for the theory which follows is the Lie algebra of all real analytic vector fields on a real analytic $n$-dimensional manifold $M^{n}$. However, we deal only with local problems in which we study the vector fields on some neighborhood $\mathcal{U}$ of a point $p \in M^{n}$. Therefore, instead of constantly referring to local coordinate charts $\chi: \mathcal{U} \rightarrow \mathbb{R}^{n}$, with $\chi(p)=0$, we will identify any point $q$ in $\mathcal{U}$ with its local coordinate expression $x=\chi(q)$ in $\chi(\mathcal{U})=\mathcal{U}$, and take the local viewpoint that our vector fields are defined on an open neighborhood $U$ of $0 \in \mathbb{R}^{n}$.

We will denote a real analytic vector field $X$, in local coordinates $x=\left(x_{1}, \ldots, x_{n}\right)$, equivalently by

$$
X(x)=\sum_{i=1}^{n} a_{i}(x) \frac{\partial}{\partial x_{i}}, \quad X(x)=\left(\begin{array}{c}
a_{1}(x) \\
\vdots \\
a_{n}(x)
\end{array}\right),
$$

where each $a_{i}(x)$ is a real analytic function of $x$.

Given two real analytic vector fields $X, Y$, we let $[X, Y]$ denote their Lie product which, in local coordinates, is expressed by $(\partial X / \partial x)(x) Y(x)-$ $(\partial Y / \partial x)(x) X(x)$, denoting by $(\partial X / \partial x)(x),(\partial Y / \partial x)(x)$ respectively the Jacobians of $X$ and $Y$. In order to simplify the notation, if $X$ and $Y$ are smooth maps from $\mathbb{R}^{n}$ to $\mathbb{R}^{n}$, we still denote by $[X, Y]$ the map defined by the above expression even in cases where we do not interpret $X, Y$ as local coordinate expressions of two vector fields. We also use $a d X(Y) \doteq(a d X, Y) \doteq[X, Y]$ and, inductively, $\left(a d^{k+1} X, Y\right) \doteq\left[X,\left(a d^{k} X, Y\right)\right]$.

If $X(x)$ denotes a vector field given in the $x$-coordinates and we perform a coordinate change $x=\phi(y)$, we denote by $T_{\phi} X(y)$ the transformed field expressed in the $y$-coordinates, which is given by $T_{\phi} X(y)=((\partial \phi / \partial y)(y))^{-1} X(\phi(y))$.

For a given vector field $X$ and $p \in \mathbb{R}^{n}$, we denote by $(\exp t X)(p)$ the solution, at time $t$, of the Cauchy problem $\dot{x}=X(x), x(0)=p$; thus the map $p \mapsto(\exp t X)(p)$, represents the flow generated by the field $X$.

Throughout it will be used the term strictly non linear to denote an analytic map whose Taylor expansion starts with a homogeneous term (with respect to the standard dilation) of degree greater than one.

The definitions of dilation and homogeneity w.r.t. a dilation have already been recalled in the introduction. In particular we will use the 
following notation. Let $\delta_{\varepsilon}^{r}: \mathbb{R}^{n} \rightarrow \mathbb{R}^{n}$ be a dilation on $\mathbb{R}^{n}, \delta_{\varepsilon}^{r}(x)=$ $\left(\varepsilon^{r_{1}} x_{1}, \ldots, \varepsilon^{r_{n}} x_{n}\right), \varepsilon>0$. The nondecreasing $n$-tuple of positive integers $r=\left(r_{1}, \ldots, r_{n}\right)$ will be said to be of the type $\left(i_{1}, \ldots, i_{m} ; j_{1}, \ldots, j_{m}\right)$ if the following equalities hold:

$$
\begin{gathered}
r_{1}=r_{2}=\ldots=r_{i_{1}}=j_{1}, \\
r_{i_{1}+1}=r_{i_{1}+2}=\ldots=r_{i_{2}}=j_{2}, \\
\vdots \quad \vdots \quad \vdots \quad \\
r_{i_{(m-1)}+1}=r_{i_{(m-1)}+2}=\ldots=r_{i_{m}}=j_{m},
\end{gathered}
$$

with $i_{m}=n$ and $j_{1}<j_{2}<\ldots<j_{m}$. (Throughout is used $i_{0}=0$.) For certain results the additional assumption $j_{1}=1$ will be required. Given a map $f: \mathbb{R}^{n} \mapsto \mathbb{R}^{n}$ with $f_{i} \in P^{r_{i}+m-1, r}\left(\mathbb{R}^{n}\right), i=1, \ldots, n$, we write $f \in H^{m, r}\left(\mathbb{R}^{n}\right)$ even in cases where $f$ does not denote the local coordinate expression of some vector field.

\subsection{Statements of the main results}

We here summarize the results presented in this section. Given a dilation $\delta_{\varepsilon}^{r}$ with $r=\left(r_{1}, \ldots, r_{n}\right)$ of the type $\left(i_{1}, \ldots, i_{m} ; j_{1}, \ldots, j_{m}\right)$ as defined in (2.1), let $Z$ denote the vector field expressed, in $x$-local coordinates, by

$$
Z(x)=\sum_{i=1}^{n} r_{i} x_{i} \frac{\partial}{\partial x_{i}}
$$

THEOREM 2.1. - Let $\phi$ be a real analytic diffeomorphism on $\mathbb{R}^{n}$ such that $\phi(0)=0$. If $j_{1}=1$ in (2.1), then the following statements are equivalent:

(i) There exists an integer $m \geq 0$ such that

$$
X \in H^{m, r}\left(\mathbb{R}^{n}\right) \Longrightarrow T_{\phi} X \in H^{m, r}\left(\mathbb{R}^{n}\right) ;
$$

(ii)

$$
T_{\phi} Z=Z
$$

(iii)

$$
\phi \in H^{1, r}\left(\mathbb{R}^{n}\right)
$$

If $j_{1}>1$ in (2.1), then (ii), (iii) are equivalent and they imply (i).

THEOREM 2.2. - A map $\phi: \mathbb{R}^{n} \rightarrow \mathbb{R}^{n}$ is a real analytic diffeomorphism, homogeneous of degree one with respect to the dilation $\delta_{\varepsilon}^{r}$, if and only if 
it has the form

$$
\phi(x)=A x+g(x),
$$

where $A$ is a matrix of the form

$$
A=\operatorname{diag}\left\{A_{1}, \ldots, A_{m}\right\},
$$

each $A_{k}$ being an invertible $\left(i_{k}-i_{k-1}\right) \times\left(i_{k}-i_{k-1}\right)$ matrix with real entries, and $g$ is a strictly non linear function in $H^{1, r}\left(\mathbb{R}^{n}\right)$ of the form

$$
g(x)=\sum_{j=2}^{j_{m}} g^{j}(x)
$$

each $g^{j}$ being homogeneous of degree $j$ with respect to the standard dilation $\delta_{\varepsilon}^{1}$.

Let $G L\left(\mathbb{R}^{n}\right)$ denote the set of all invertible linear transformations on $\mathbb{R}^{n}$. We will sometimes identify the elements of $G L\left(\mathbb{R}^{n}\right)$ with their matrix representation, denoting by $A$ the linear transformation $x \mapsto A x$. Consider the sets of transformations

$$
\begin{gathered}
G_{1}=\left\{A \in G L\left(\mathbb{R}^{n}\right): \text { A has the form }(2.4)\right\}, \\
G_{2}=\left\{\phi \in H^{1, r}\left(\mathbb{R}^{n}\right): \phi=I+g\right\}, \\
G H^{1, r}\left(\mathbb{R}^{n}\right)=\left\{\phi \in H^{1, r}\left(\mathbb{R}^{n}\right): \phi=A+g, \text { A is invertible }\right\},
\end{gathered}
$$

(here $I$ denotes as usual the identity matrix, and $g$ the strictly non linear part of $\phi$ ). From Theorems 2.1 and 2.2 it follows that, in the case $\delta_{\varepsilon}^{r}$, $r=\left(r_{1}, \ldots, r_{n}\right)$, is a dilation with $r_{1}=1, G H^{1, r}\left(\mathbb{R}^{n}\right)$ coincides with the set of all real analytic diffeomorphisms that transform elements of $H^{m, r}\left(\mathbb{R}^{n}\right)$ into elements of the same space.

THEOREM 2.3. - The set $G H^{1, r}\left(\mathbb{R}^{n}\right)$ is a finite dimensional Lie group with the dimension depending on $r=\left(r_{1}, \ldots r_{n}\right)$. Moreover, the sets $G_{1}, G_{2}$ defined above are subgroups of $G H^{1, r}\left(\mathbb{R}^{n}\right)$, and for any $\phi \in G H^{1, r}\left(\mathbb{R}^{n}\right)$ there exist $\phi_{1} \in G_{1}$ and $\phi_{2}, \psi_{2} \in G_{2}$, such that

$$
\phi=\phi_{1} \circ \phi_{2}=\psi_{2} \circ \phi_{1}
$$

Remark 2.4. - The group $G H^{1, r}\left(\mathbb{R}^{n}\right)$ is the natural generalization of the group of the invertible linear transformations $G L\left(\mathbb{R}^{n}\right)$, with which it coincides in the case $\delta_{\varepsilon}^{r}$ is the standard dilation $\delta_{\varepsilon}^{1}$. 
THEOREM 2.5. - Let $X \in H^{1, r}\left(\mathbb{R}^{n}\right)$. Then, for each fixed $t \in \mathbb{R}$, the map

$$
p \rightarrow(\operatorname{expt} X)(p), \quad p \in \mathbb{R}^{n}
$$

is a homogeneous analytic diffeomorphism of degree one with respect to $\delta_{\varepsilon}^{r}$. Moreover, $H^{1, r}\left(\mathbb{R}^{n}\right)$ is the Lie algebra of the Lie group $G H^{1, r}\left(\mathbb{R}^{n}\right)$.

Example 2.6. - On $\mathbb{R}^{2}$ let $X(x)=2 x_{1} \partial / \partial x_{1}+\left(5 x_{1}^{3}-x_{2}\right) \partial / \partial x_{2}$. Note that $X$ is a field in $H^{1, r}\left(\mathbb{R}^{2}\right)$, with $r=(1,3)$. It can be easily computed that the flow-map of $X$ is given by $(\exp t X)\left(p_{1}, p_{2}\right)=$ $\left(e^{2 t} p_{1},(5 / 7)\left(e^{6 t}-e^{-t}\right) p_{1}^{3}+e^{-t} p_{2}\right)$, which is clearly an element of $G H^{1, r}\left(\mathbb{R}^{2}\right)$ for any fixed $t \in \mathbb{R}$.

\subsection{Preliminary lemmas}

We collect here several preliminary results that will enable us to prove Theorem 2.1.

LEMMA 2.7. - Let $Z$ be as in (2.2). A real analytic vector field $X(x)=\sum_{i=1}^{n} a_{i}(x) \partial / \partial x_{i}$ is homogeneous of degree $m$ with respect to $\delta_{\varepsilon}^{r}$ if and only if

$$
[X, Z]=(m-1) X
$$

Proof. - The $i$-th component of the field $[X, Z]$ is given by

$$
\sum_{j=1}^{n} \frac{\partial a_{i}}{\partial x_{j}}(x) r_{j} x_{j}-r_{i} a_{i}(x)
$$

Since $X$ is analytic, its components $a_{i}$ can be expanded in terms of homogeneous polynomials; let $\bar{a}_{i}$ denote a monomial of $a_{i}$ of the form $\bar{a}_{i}(x)=a x_{1}^{\nu_{1}} \cdot \cdots \cdot x_{n}^{\nu_{n}}$. Then we have

$$
\sum_{j=1}^{n} \frac{\partial \bar{a}_{i}}{\partial x_{j}}(x) r_{j} x_{j}=\sum_{j=1}^{n} \frac{\bar{a}_{i}(x)}{x_{j}} \nu_{j} r_{j} x_{j}=\left(\sum_{j=1}^{n} \nu_{j} r_{j}\right) \bar{a}_{i}(x)
$$

Substituting the above in (2.8), for any monomial $\bar{a}_{i}$ of $a_{i}$, we deduce that the relation (2.7) holds if and only if the exponents $\nu_{1}, \ldots, \nu_{n}$ of any monomial $\bar{a}_{i}(x)=a x_{1}^{\nu_{1}} \cdot \cdots \cdot x_{n}^{\nu_{n}}$ of $a_{i}$, satisfy the relation

$$
\sum_{j=1}^{n} \nu_{j} r_{j}-r_{i}=m-1
$$


for each $i=1, \ldots, n$. This means that $a_{i} \in P^{r_{i}+m-1, r}\left(\mathbb{R}^{n}\right)$, for each $i=1, \ldots, n$ which is equivalent to say that $X \in H^{m, r}\left(\mathbb{R}^{n}\right)$.

LEMMA 2.8. - Let $X$ be a real analytic vector field on $\mathbb{R}^{n}$, homogeneous of degree $m$ with respect to $\delta_{\varepsilon}^{r}$. If $\phi$ is a real analytic diffeomorphism such that

$$
T_{\phi} Z=Z
$$

then also $T_{\phi} X$ is an homogeneous field of degree $m$ with respect to $\delta_{\varepsilon}^{r}$.

Proof. - Recall that the Lie product is a coordinate-free operation; thus, transforming both sides of (2.7), we obtain

$$
T_{\phi}[X, Z]=\left[T_{\phi} X, T_{\phi} Z\right]=(m-1) T_{\phi} X
$$

Hence, substituting (2.9) into (2.10), we have $\left[T_{\phi} X, Z\right]=(m-1) T_{\phi} X$ which, using lemma 2.7 , enables us to conclude.

LEMMA 2.9. - Let $Y(x)=\sum_{i=1}^{n} a_{i}(x) \partial / \partial x_{i}$ be a non-zero real analytic homogeneous vector field of degree $j \in \mathbb{Z}$ with respect to $\delta_{\varepsilon}^{r}, r=\left(r_{1}, \ldots, r_{n}\right)$, with $r_{1}=1$. Then

$$
[X, Y]=0 \text { for all } X \in H^{m, r}\left(\mathbb{R}^{n}\right),
$$

for some $m \geq 0$, if and only if one of the following two conditions holds

(i) $\delta_{\varepsilon}^{r}=\delta_{\varepsilon}^{1}, \quad m=0, \quad Y(x)=\sum_{i=1}^{n} a_{i} \partial / \partial x_{i}, \quad a_{i} \in \mathbb{R}$;

(ii) $m=1, \quad Y(x)=a Z(x), \quad a \in \mathbb{R}$.

Proof. - That (i) implies (2.11) is immediate since an homogeneous vector field of degree zero with respect to the standard dilation is a constant vector field and the Lie bracket of two constant fields is clearly zero.

Next suppose (ii); from lemma 2.7, using the linearity of the Lie product, it follows

$$
[X, a Z]=a(m-1) X, \text { for any } X \in H^{m, r}\left(\mathbb{R}^{n}\right) .
$$

Therefore, since $m=1,(2.11)$ is satisfied.

Now suppose that $(2.11)$ holds for some $m \geq 0$. Note first that, since $m \geq 0$ implies $r_{k}+m-1 \geq 0, k=1, \ldots, n$, then $\left\{x_{1}^{r_{k}+m-1} \partial / \partial x_{k}, k=\right.$ $1, \ldots, n\}$ is a set of non zero fields in $H^{m, r}\left(\mathbb{R}^{n}\right)$. Therefore $(2.11)$ is in 
particular satisfied by the elements of this set:

$$
\begin{aligned}
0 & =\left[\begin{array}{l}
\left.x_{1}^{r_{k}+m-1} \partial / \partial x_{k}, Y\right] \\
-\frac{\partial a_{1}}{\partial x_{k}}(x) x_{1}^{r_{k}+m-1} \\
\vdots \\
\left(r_{k}+m-1\right) x_{1}^{r_{k}+m-2} a_{1}(x)-\frac{\partial a_{k}}{\partial x_{k}}(x) x_{1}^{r_{k}+m-1} \\
\vdots \\
-\frac{\partial a_{n}}{\partial x_{k}}(x) x_{1}^{r_{k}+m-1} \\
k=1, \ldots n .
\end{array}\right)
\end{aligned}
$$

Thus it follows

$$
\frac{\partial a_{i}}{\partial x_{k}}(x) \equiv 0 \quad i, k=1, \ldots, n, \quad i \neq k,
$$

which implies, using $Y \in H^{j, r}\left(\mathbb{R}^{n}\right)$ and therefore $a_{i} \in P^{r_{i}+j-1, r}\left(\mathbb{R}^{n}\right)$,

$$
a_{i}(x)=a_{i} x_{i}^{\nu_{i}}, \quad a_{i} \in \mathbb{R}, \quad r_{i} \nu_{i}=r_{i}+j-1, \quad i=1, \ldots, n .
$$

We now consider three cases:

Case 1. - Suppose $a_{1}=0$. From (2.12) it follows $\left.\left(\partial a_{k} / \partial x_{k}\right)(x)\right) \equiv 0$, for each $k=1, \ldots, n$ and so $\nu_{i}=0$ for each $i=1, \ldots, n$ in (2.14), which implies $r_{i}=1$, for each $i=1, \ldots, n$, and $j=0$. Thus condition (i) is satisfied.

Case 2. - Suppose $a_{1} \neq 0, \nu_{k}=0$ for some $k, 1 \leq k \leq n$. Then $m \geq 0$ and (2.12) imply $r_{k}=1, m=0$. Hence $r_{i}=1$ for each $i=1, \ldots, k$ which, using again (2.12), implies $\left.\left(\partial a_{i} / \partial x_{i}\right)(x)\right) \equiv 0$, for each $i=1, \ldots, k$. Thus $a_{i}(x) \equiv a_{i} \in \mathbb{R}, \nu_{i}=0$, for each $i=1, \ldots, k$ and $j=0$. If $\nu_{i}>0$ for some $i>k$, then, using (2.14), we would have $j=r_{i}\left(\nu_{i}-1\right)+1 \geq 1$ which gives a contradiction. Hence $\nu_{i}=0$ for each $i=1, \ldots, n$ and condition (i) is satisfied.

Case 3. - Suppose $a_{1} \neq 0, \nu_{i}>0$ for each $i=1, \ldots, n$. From (2.12), using (2.14), it follows

$$
\begin{array}{r}
0=\left(r_{k}+m-1\right) x_{1}^{r_{k}+m-2} a_{1} x_{1}^{\nu_{1}}-a_{k} \nu_{k} x_{k}^{\nu_{k}-1} x_{1}^{r_{k}+m-1}, \\
k=1, \ldots, n,
\end{array}
$$


which implies $\nu_{k}=1$ for each $k=2, \ldots, n$, and so by (2.14) $j=1$. Moreover, since $r_{1}=1$, (2.14) implies also $\nu_{1}=j$. Thus $\nu_{i}=1$ for each $i=1, \ldots n$. Finally (2.15), with $k=1$, implies $m=1$, and, with $k=2, \ldots, n$, implies $a_{k}=r_{k} a_{1}$ for each $k=2, \ldots, n$. Hence condition (ii) is satisfied with $a=a_{1}$.

LEMMA 2.10. - Let $Y(x)=\sum_{i=1}^{n} a_{i}(x) \partial / \partial x_{i}$ be a non-zero real analytic vector field on $\mathbb{R}^{n}$. Then the same conclusions of lemma 2.9 hold.

Proof. - First expand $Y$ in homogeneous vector fields with respect to $\delta_{\varepsilon}^{r}$, i.e.,

$$
Y(x)=\sum_{j=1-r_{n}}^{\infty} Y^{j}(x), \quad Y^{j} \in H^{j, r}\left(\mathbb{R}^{n}\right) .
$$

Next observe that, since $\left[X, Y^{j}\right], j \geq 1-r_{n}$, are homogeneous vector fields of different degrees, the equation

$$
0=[X, Y]=\sum_{j=1-r_{n}}^{\infty}\left[X, Y^{j}\right], \text { for any } X \in H^{m, r}\left(\mathbb{R}^{n}\right)
$$

is satisfied if and only if the equations

$$
\left[X, Y^{j}\right]=0, \text { for any } j \geq 1-r_{n}, X \in H^{m, r}\left(\mathbb{R}^{n}\right),
$$

are satisfied simultaneously. Hence we can apply lemma 2.9 and obtain the same conclusion.

LEMMA 2.11. - Let $\phi$ be a real analytic diffeomorphism on $\mathbb{B}^{n}$ such that $\phi(0)=0$. Assume that $j_{1}=1$ in (2.1). If

$$
X \in H^{m, r}\left(\mathbb{R}^{n}\right) \Longrightarrow T_{\phi} X \in H^{m, r}\left(\mathbb{R}^{n}\right)
$$

for some $m \geq 0$, then

$$
T_{\phi} Z=Z
$$

Proof. - By lemma 2.7 we know that (2.16) implies

$$
\left[T_{\phi} X, Z\right]=(m-1) T_{\phi} X \text { for any } X \in H^{m, r}\left(\mathbb{R}^{n}\right) .
$$

Transforming both sides of (2.18) under the action of the map $T_{\phi^{-1}}$, which is the inverse map of $T_{\phi}$, we obtain

$$
\left[X, T_{\phi^{-1}} Z\right]=(m-1) X \text { for any } X \in H^{m, r}\left(\mathbb{R}^{n}\right)
$$


which, together with (2.7), implies

$$
\left[X, T_{\phi^{-1}} Z-Z\right]=0, \text { for any } X \in H^{m, r}\left(\mathbb{R}^{n}\right) .
$$

We now distinguish three cases:

Case 1. - Suppose $\delta_{\varepsilon}^{r}=\delta_{\varepsilon}^{1}, m=0$. By lemma 2.10 it follows

$$
T_{\phi-1} Z=\sum_{i=1}^{n} a_{i} \frac{\partial}{\partial x_{i}}+Z, \quad a_{i} \in \mathbb{R} .
$$

that can be explicitly written as

$$
\left(\frac{\partial \phi^{-1}}{\partial x}(x)\right)^{-1}\left(\begin{array}{c}
\phi_{1}^{-1}(x) \\
\vdots \\
\phi_{n}^{-1}(x)
\end{array}\right)=\left(\begin{array}{c}
a_{1} \\
\vdots \\
a_{n}
\end{array}\right)+\left(\begin{array}{c}
x_{1} \\
\vdots \\
x_{n}
\end{array}\right)
$$

If we evaluate the above at $x=0$ and observe that $\phi(0)=0$ implies $\phi^{-1}(0)=0$, we obtain $a_{i}=0, i=1, \ldots, n$. Hence $T_{\phi^{-1}} Z=Z$ which implies (2.17).

Case 2. - Suppose $m=1$. From (2.19), using lemma 2.10, it follows

$$
T_{\phi^{-1}} Z=(a+1) Z \text {, }
$$

for some $a \in \mathbb{R} \backslash\{-1\}\left(a \neq-1\right.$ since $T_{\phi^{-1}} Z=0$ implies $Z=0$, by applying $T_{\phi}$ to $\left.(2.21)\right)$. Set $b=(a+1)^{-1}$. Then, using the linearity of $T_{\phi}$, (2.21) implies $T_{\phi} Z=b Z, b \in \mathbb{R} \backslash\{0\}$, from which it follows

$$
\left(\begin{array}{c}
r_{1} \phi_{1}(x) \\
\vdots \\
r_{n} \phi_{n}(x)
\end{array}\right)=\left(\frac{\partial \phi}{\partial x}(x)\right)\left(\begin{array}{c}
b r_{1} x_{1} \\
\vdots \\
b r_{n} x_{n}
\end{array}\right)
$$

Since $\phi$ is analytic, its components $\phi_{i}$ can be expanded in terms of homogeneous polynomials; let $\bar{\phi}_{i}$ denote a monomial of $\phi_{i}$ of the form $\bar{\phi}_{i}(x)=\alpha x_{1}^{\nu_{1}} \cdot \ldots \cdot x_{n}^{\nu_{n}}$. Then (2.22) implies

$$
r_{i} \bar{\phi}_{i}(x)=\sum_{k=1}^{n} \frac{\bar{\phi}_{i}(x)}{x_{k}} \nu_{k} b r_{k} x_{k}=b\left(\sum_{k=1}^{n} \nu_{k} r_{k}\right) \bar{\phi}_{i}(x) \text {. }
$$

Thus (2.22) is equivalent to the condition that the exponents $\nu_{1}, \ldots, \nu_{n}$ of all the monomials $\bar{\phi}_{i}(x)=\alpha x_{1}^{\nu_{1}} \cdot \ldots \cdot x_{n}^{\nu_{n}}$ of the same $i$-th component $\phi_{i}$ of $\phi$, must satisfy the relation

$$
r_{i}=b\left(\sum_{k=1}^{n} \nu_{k} r_{k}\right)
$$


for each $i=1, \ldots, n$. This relation implies $b>0$ and

$$
\phi_{i} \in P^{r_{i}+l_{i}-1, r}\left(\mathbb{R}^{n}\right),
$$

for each $i=1, \ldots, n$, with $l_{i}$ integers satisfying

$$
r_{i}=b\left(r_{i}+l_{i}-1\right), \quad i=1, \ldots, n .
$$

In particular (2.25), evaluated for $i=1$, gives $l_{1}=b^{-1}$ and thus, by substituting it back in (2.25), we have

$$
r_{i}+l_{i}-1=r_{i} l_{1}, \quad i=1, \ldots, n .
$$

Note that from the above it follows that $l_{1}$ is a positive integer since $b>0$. Moreover, if $l_{1}>1$, from (2.24) and (2.26) we have

$$
\phi_{n} \in P^{r_{n} l_{1}, r}\left(\mathbb{R}^{n}\right), \quad r_{n} l_{1}>r_{n},
$$

which implies that the $n$-th component of $\phi$ is the sum of homogeneous terms of degree greater than one with respect to the standard dilation. But this would imply $\operatorname{det}((\partial \phi / \partial x)(0))=0$ which cannot be since $\phi$ is a diffeomorphism. Thus $l_{1}=1$ which implies $b=1$ and $a=0$ in (2.21), from which (2.17) follows.

Case 3. - Suppose $\delta_{\varepsilon}^{r}=\delta_{\varepsilon}^{1}, m>1$, or $\delta_{\varepsilon}^{r} \neq \delta_{\varepsilon}^{1}, m \neq 1$. From (2.19), using lemma 2.10, it follows that $T_{\phi^{-1}} Z-Z=0$ and thus (2.17).

COROLLARY 2.12. - Let $\phi$ be a real analytic diffeomorphism on $\mathbb{R}^{n}$ such that $\phi(0)=0$. Then the following statements are equivalent:

(i) $T_{\phi} Z=Z$;

(ii) $\phi \in H^{1, r}\left(\mathbb{R}^{n}\right)$.

Proof. - First suppose that (i) is satisfied. We have shown, in the proof of case 2 of lemma 2.11 , that this condition, which is equivalent to (2.22) with $b=1$, implies (2.24). Then, using (2.25) with $b=1$, it follows that $\phi_{i} \in P^{r_{i}, r}\left(\mathbb{R}^{n}\right)$, for each $i=1, \ldots, n$ and so $\phi \in H^{1, r}\left(\mathbb{R}^{n}\right)$.

Next suppose that (ii) is satisfied. This condition implies that (2.23), in the proof of case 2 of lemma 2.11 , is satisfied with $b=1$ by any monomial $\bar{\phi}_{i}$ of each component $\phi_{i}$ of $\phi$. It follows (2.22) with $b=1$, which is equivalent to condition (i).

\subsection{Proofs of the main results}

Proof of Theorem 2.1. - We need only to observe that: 
if $j_{1}=1$, condition (i) implies (ii) by lemma 2.11;

if $j_{1} \geq 1$, condition (ii) implies (i) by lemma 2.8 ;

if $j_{1} \geq 1$, condition (ii) is equivalent to (iii) by Corollary 2.12 .

Proof of Theorem 2.2. - From the definition of homogeneity with respect to a dilation, it follows immediately that $\phi$ is a real analytic homogeneous function of degree one with respect to $\delta_{\varepsilon}^{r}$ if and only if it has the form (2.3) - (2.5), with $A_{k}$ generic $\left(i_{k}-i_{k-1}\right) \times\left(i_{k}-i_{k-1}\right)$ matrices with real entries. Thus we need only to show that, under the hypothesis $\phi \in H^{1, r}\left(\mathbb{R}^{n}\right)$, the matrices $A_{k}$ in (2.4) are invertible if and only if $\phi$ is a diffeomorphism on $\mathbb{R}^{n}$.

One implication is obvious since if $\phi$ is a diffeomorphism then the Jacobian $(\partial \phi / \partial x)(0)$ is invertible and, from (2.3), it is clear that $(\partial \phi / \partial x)(0)=A$ which, having the form (2.4), is invertible if and only if each block $A_{k}$ is invertible, $k=1, \ldots, m$.

Suppose now that $A_{k}$ in (2.4) are invertible matrices for each $k=$ $1, \ldots, m$. We will show that $\phi$ has a continuously differentiable inverse map on $\mathbb{R}^{n}$. Define the map

$$
\psi: \mathbb{R}^{n} \rightarrow \mathbb{R}^{n}, \quad \psi(x)=A^{-1} x+h(x),
$$

where $h$ is a function whose $i$-th component is recursively defined by

$$
\begin{aligned}
& h_{i}(x)=0, \quad i=1, \ldots i_{1} \text {; } \\
& \left(\begin{array}{c}
h_{i_{k-1}+1}(x) \\
\vdots \\
h_{i_{k}}(x)
\end{array}\right)= \\
& -A_{k}^{-1}\left(\begin{array}{ccc}
g_{i_{k-1}+1} & \left(\left(\begin{array}{ccc}
A_{1}^{-1} & & \\
& \ddots & \\
& & A_{k-1}^{-1}
\end{array}\right)\left(\begin{array}{c}
x_{1} \\
\vdots \\
x_{i_{k-1}}
\end{array}\right)+\left(\begin{array}{c}
h_{1}(x) \\
\vdots \\
\vdots \\
h_{i_{k-1}}(x)
\end{array}\right)\right) \\
g_{i_{k}} & \left(\left(\begin{array}{ccc}
A_{1}^{-1} & \vdots & \\
& \ddots & \\
& & A_{k-1}^{-1}
\end{array}\right)\left(\begin{array}{c}
x_{1} \\
\vdots \\
x_{i_{k-1}}
\end{array}\right)+\left(\begin{array}{c}
h_{1}(x) \\
\vdots \\
h_{i_{k-1}}(x)
\end{array}\right)\right)
\end{array}\right) \\
& k=2, \ldots, m \text {. }
\end{aligned}
$$

Note that this definition makes sense since the fact that $g$ is a strictly non linear map in $H^{1, r}\left(\mathbb{R}^{n}\right)$ implies that the components $g_{i}, i=i_{k-1}+1, \ldots, i_{k}$ are functions depending only on the previous $i_{k-1}$ variables $\left(x_{1}, \ldots x_{i_{k-1}}\right)$. 
Thus it can be easily checked, by recursion on $k=1, \ldots m$, that

$$
\left(\begin{array}{c}
(\phi \circ \psi)_{i_{k-1}+1}(x) \\
\vdots \\
(\phi \circ \psi)_{i_{k}}(x)
\end{array}\right)=\left(\begin{array}{c}
(\psi \circ \phi)_{i_{k-1}+1}(x) \\
\vdots \\
(\psi \circ \phi)_{i_{k}}(x)
\end{array}\right)=\left(\begin{array}{c}
x_{i_{k-1}+1} \\
\vdots \\
x_{i_{k}}
\end{array}\right) .
$$

Hence $\psi=\phi^{-1}$ and, from definition (2.27), (2.28), it is clear that $\psi$ is continuously differentiable, thus concluding the proof.

Proof of Theorem 2.3. - By Theorem 2.2 and Corollary 2.12 we know that $\phi \in G H^{1, r}\left(\mathbb{R}^{n}\right)$ if and only if $\phi$ is a diffeomorphism that fixes zero such that $T_{\phi} Z=Z$. Thus for $\phi \in G H^{1, r}\left(\mathbb{R}^{n}\right)$, from

$$
Z=T_{i d} Z=T_{\phi^{-1} \circ \phi} Z=T_{\phi^{-1}} T_{\phi} Z=T_{\phi^{-1}} Z
$$

it follows that the inverse $\phi^{-1}$ is an element of $G H^{1, r}\left(\mathbb{R}^{n}\right)$. Also we have, using the definition of homogeneity of degree one with respect to a dilation,

$$
\phi \circ \psi\left(\delta_{\varepsilon}^{r}(x)\right)=\phi\left(\psi\left(\delta_{\varepsilon}^{r}(x)\right)\right)=\phi\left(\delta_{\varepsilon}^{r}(\psi(x))\right)=\delta_{\varepsilon}^{r}(\phi(\psi(x)))
$$

for any $\phi, \psi \in H^{1, r}\left(\mathbb{R}^{n}\right)$. This shows that $G H^{1, r}\left(\mathbb{R}^{n}\right)$ is a group. The fact that $G_{1}, G_{2}$ are subgroups of $G H^{1, r}\left(\mathbb{R}^{n}\right)$ is an immediate consequence of their definitions and of the form (2.27), (2.28) of the inverse map of an element in $G H^{1, r}\left(\mathbb{R}^{n}\right)$. Let now $\phi$ be an element of $G H^{1, r}\left(\mathbb{R}^{n}\right)$ of the form (2.3) - (2.5), $\phi(x)=A x+g(x)$, and consider the following maps

$$
\begin{aligned}
& \phi_{1}(x)=A x, \\
& \phi_{2}(x)=x+A^{-1} g(x), \\
& \psi_{2}(x)=x+g\left(A^{-1} x\right) .
\end{aligned}
$$

Note that $A^{-1} \circ g$ and $g \circ A^{-1}$ are compositions of two elements of $H^{1, r}\left(\mathbb{R}^{n}\right)$ and therefore still elements of $H^{1, r}\left(\mathbb{R}^{n}\right)$ by the previous argument; also they are strictly non linear maps since $g$ is. Hence $\phi_{1} \in G_{1}$ and $\phi_{2}, \psi_{2} \in G_{2}$ and the equality (2.6) is clearly satisfied.

Finally, to prove that $G H^{1, r}\left(\mathbb{R}^{n}\right)$ is a Lie group, observe that $H^{1, r}\left(\mathbb{R}^{n}\right)$ is a finite dimensional analytic manifold isomorphic to $\mathbb{R}^{p_{r}}$, with $p_{r}$ a constant depending on $r=\left(r_{1}, \ldots, r_{n}\right)$ (more precisely on the $2 m$ tuple $\left(i_{1}, \ldots, i_{m} ; j_{1}, \ldots j_{m}\right)$ associated to $r$ in $\left.(2.1)\right)$. Note that $G H^{1, r}\left(\mathbb{R}^{n}\right)$ coincides with the open subset $\left\{\phi \in H^{1, r}\left(\mathbb{R}^{n}\right): \phi=A+g, \operatorname{det} A \neq 0\right\}$ of $H^{1, r}\left(\mathbb{R}^{n}\right)$. Thus also $G H^{1, r}\left(\mathbb{R}^{n}\right)$ is a $p_{r}$ dimensional analytic manifold and the group operation (the composition of maps) and the inversion $\left(i: \phi \rightarrow \phi^{-1}\right)$ are clearly analytic. 
Proof of Theorem 2.5. - We first observe that, by Theorem 4.3 in Section 4 , the flow $(\exp t X)(x), x \in \mathbb{R}^{n}$, generated by the field $X$ is defined for all $t \in \mathbb{R}$. Let $Z$ be the vector field defined in (2.2). It can be easily verified that

$$
\delta_{\varepsilon}^{r}(x)=(\exp (\ln \varepsilon) Z)(x), \quad x \in \mathbb{R}^{n}, \quad \varepsilon>0
$$

Since, by Lemma $2.7, X \in H^{1, r}\left(\mathbb{R}^{n}\right)$ implies $[X, Z]=0$, it follows that, for any fixed $t \in \mathbb{R}$,

$$
\begin{aligned}
\delta_{\varepsilon}^{r}((\operatorname{exptX})(x)) & =(\exp (\ln \varepsilon) Z) \circ(\operatorname{exptX})(x) \\
& =(\exp t X) \circ(\exp (\ln \varepsilon) Z)(x) \\
& =(\exp t X)\left(\delta_{\varepsilon}^{r}(x)\right), \quad x \in \mathbb{R}^{n}
\end{aligned}
$$

which shows the homogeneity of the map $x \rightarrow(\exp t X)(x), t$ fixed. Moreover, it is well known from the theory of differential equations that the map $x \rightarrow(\exp t X)(x)$ is analytic and therefore we can conclude that it is an element of $H^{1, r}\left(\mathbb{R}^{n}\right)$. To show that $H^{1, r}\left(\mathbb{R}^{n}\right)$ is the Lie algebra of the Lie group $G H^{1, r}\left(\mathbb{R}^{n}\right)$, we observe that, by Theorem 2.2, $G H^{1, r}\left(\mathbb{R}^{n}\right)$ is the group of the invertible elements of the associative algebra $H^{1, r}\left(\mathbb{R}^{n}\right)$ (with the composition of functions as multiplication). Thus, the conclusion follows from a general result in the theory of Lie algebras (see $[24$, Section 2.3]).

\section{DECOMPOSITION OF HOMOGENEOUS VECTOR FIELDS OF DEGREE ONE WITH RESPECT TO AN ARBITRARY DILATION}

\subsection{Proof of the main result}

Before giving the proof of Theorem 3.1, we want to observe that the decomposition of vector fields given in this theorem would be an immediate consequence of a classical result in the theory of Lie algebras (the Jordan decomposition for elements of finite dimensional, semisimple Lie algebras: see [24, Thm. 3.10.6] ), if $H^{1, r}\left(\mathbb{R}^{n}\right)$ or, at least $H^{1, r}\left(\mathbb{R}^{n}\right) / C\left(H^{1, r}\left(\mathbb{R}^{n}\right)\right)\left(C\left(H^{1, r}\left(\mathbb{R}^{n}\right)\right)\right.$ denoting the center of $\left.H^{1, r}\left(\mathbb{R}^{n}\right)\right)$, would have been semisimple. Nevertheless it can be easily seen that this is not the case for any dilation $\delta_{\varepsilon}^{r}$ different from the standard dilation $\delta_{\varepsilon}^{1}$.

Recall that a Lie algebra is said to be semisimple if it does not possess any non zero solvable ideal. From lemma 2.9 it follows that $C\left(H^{1, r}\left(\mathbb{R}^{n}\right)\right)=\{a Z(x): a \in \mathbb{R}\}$. Thus, since the center is a non zero abelian (thus solvable) ideal, it is clear that $H^{1, r}\left(\mathbb{R}^{n}\right)$ is not semisimple. 
Regarding $H^{1, r}\left(\mathbb{R}^{n}\right) / C\left(H^{1, r}\left(\mathbb{R}^{n}\right)\right)$, if $\delta_{\varepsilon}^{r} \neq \delta_{\varepsilon}^{1}$ we can consider the subspace $\mathcal{G}$ that is generated by the following set

$$
\left\{x^{\nu} \frac{\partial}{\partial x_{i}}+C\left(H^{1, r}\left(\mathbb{R}^{n}\right)\right): x^{\nu} \frac{\partial}{\partial x_{i}} \in H^{j_{m}, 1}\left(\mathbb{R}^{n}\right) \cap H^{1, r}\left(\mathbb{R}^{n}\right)\right\} .
$$

Note that, if $x^{\alpha} \partial / \partial x_{s}$ is an element in $H^{j, 1}\left(\mathbb{R}^{n}\right) \cap H^{1, r}\left(\mathbb{R}^{n}\right), j \in$ $\left\{j_{1}, \ldots, j_{m}\right\}$, we have

$$
\left[x^{\nu} \frac{\partial}{\partial x_{i}}, x^{\alpha} \frac{\partial}{\partial x_{s}}\right] \in H^{j+j_{m}-1, \mathbf{1}}\left(\mathbb{R}^{n}\right) \cap H^{1, r}\left(\mathbb{R}^{n}\right) .
$$

But, since $H^{k, 1}\left(\mathbb{R}^{n}\right) \cap H^{1, r}\left(\mathbb{R}^{n}\right)=\{0\}$, for any $k>j_{m}$, it follows that the above product, if not zero, must be an element of $H^{j_{m}, 1}\left(\mathbb{R}^{n}\right) \cap H^{1, r}\left(\mathbb{R}^{n}\right)$. Thus it can be easily seen that $\mathcal{G}$ is an ideal of $H^{1, r}\left(\mathbb{R}^{n}\right) / C\left(H^{1, r}\left(\mathbb{R}^{n}\right)\right.$. Moreover, observing again that the Lie product of two elements in $H^{j_{m}, 1}\left(\mathbb{R}^{n}\right) \cap H^{1, r}\left(\mathbb{R}^{n}\right)$ is an element of $H^{2 j_{m}-1,1}\left(\mathbb{R}^{n}\right) \cap H^{1, r}\left(\mathbb{R}^{n}\right)$, we deduce that their product is zero since $j_{m}>1$ implies $2 j_{m}-1>j_{m}$. Therefore $\mathcal{G}$ is an abelian ideal of $H^{1, r}\left(\mathbb{R}^{n}\right) / C\left(H^{1, r}\left(\mathbb{R}^{n}\right)\right.$ that cannot be semisimple.

Proof of Theorem 3.1. - Since $X \in H^{1, r}\left(\mathbb{R}^{n}\right)$, its expansion in homogeneous fields with respect to the standard dilation has the form

$$
X(x)=A x+\sum_{j=2}^{j_{m}} X^{j}(x), \quad X^{j} \in H^{j, 1}\left(\mathbb{R}^{n}\right) \cap H^{1, r}\left(\mathbb{R}^{n}\right) .
$$

We know that, if $S$ and $\widehat{N}$ denote the semisimple and nilpotent part of the linear field $A$, we have $[S, \widehat{N}]=0$. Thus, in order to obtain the decomposition (1.3) satisfying (1.4), we will look for coordinate transformations that leave unchanged the linear part of $X$ and remove, from the non linear part, terms of increasing degree not commuting with the semisimple field $S$. More precisely we will show, using induction, that there exist a finite sequence of transformations $\phi_{k} \in G H^{1, r}\left(\mathbb{R}^{n}\right)$, $1 \leq k \leq j_{m}$, satisfying

$$
\begin{aligned}
T_{\phi_{1}} X=X, & \\
T_{\phi_{k}} T_{\phi_{1} \circ \ldots \circ \phi_{k-1}} X= & \sum_{j=1}^{k-1}\left(T_{\phi_{1} \circ \ldots \circ \phi_{k-1}} X\right)^{j}+\sum_{j=k}^{j_{m}} Y^{j}, \\
& {\left[S, \sum_{j=2}^{k-1}\left(T_{\phi_{1} \circ \ldots \circ \phi_{k-1}} X\right)^{j}+Y^{k}\right]=0, \quad k>1, }
\end{aligned}
$$


where $\left(T_{\phi_{1} \circ \ldots \circ \phi_{k-1}} X\right)^{j}$ denotes the homogeneous part of degree $j$ (with respect to the standard dilation) of the field $X$ after performing the transformation $x=\phi_{1} \circ \ldots \circ \phi_{k-1}(y)$, and $Y^{j} \in H^{j, 1}\left(\mathbb{R}^{n}\right) \cap H^{1, r}\left(\mathbb{R}^{n}\right)$.

Since we know, from Theorem 2.3, that the set $G H^{1, r}\left(\mathbb{R}^{n}\right)$ is a group, the composition of these transformations is also an element of $G H^{1, r}\left(\mathbb{R}^{n}\right)$ and therefore their product $\phi=\phi_{1} \circ \ldots \circ \phi_{j_{m}}$ will produce a change of coordinates in $G H^{1, r}\left(\mathbb{R}^{n}\right)$ that gives $X$ the form (1.3), (1.4), thus proving the theorem.

It is clear that the map $\phi_{1}=I$ is an element of $G H^{1, r}\left(\mathbb{R}^{n}\right)$ that satisfies (3.2). Next suppose $\phi_{s} \in G H^{1, r}\left(\mathbb{R}^{n}\right), 1 \leq s<k, k>1$, satisfying (3.3) have already been constructed. Since $\phi_{s} \in G H^{1, r}\left(\mathbb{R}^{n}\right)$, it follows from Theorem 2.1 that $T_{\phi_{1} \circ \ldots \circ \phi_{s}} X \in H^{1, r}\left(\mathbb{R}^{n}\right)$, for all $1 \leq s<k$. This, together with the inductive assumption, implies that $T_{\phi_{1} \circ \ldots \circ \phi_{k-1}} X$ is a field of the form

$$
A+\sum_{j=2}^{j_{m}} \tilde{X}^{j}, \quad\left[S, \sum_{j=2}^{k-1} \tilde{X}^{j}\right]=0, \quad \tilde{X}^{j} \in H^{j, 1}\left(\mathbb{R}^{n}\right) \cap H^{1, r}\left(\mathbb{R}^{n}\right) .
$$

Suppose that the field $T_{\phi_{1} \circ \ldots \circ \phi_{k-1}} X$ is given in the $x$-coordinates and consider the coordinate transformation $x=\phi_{k}(y)$, with

$$
\phi_{k}=I+g^{k}, \quad g^{k} \in H^{k, 1}\left(\mathbb{R}^{n}\right) \cap H^{1, r}\left(\mathbb{R}^{n}\right)
$$

The field (3.4), expressed in the new $y$-coordinates, becomes

$$
\left(I+\frac{\partial g^{k}}{\partial y}(y)\right)^{-1}\left(A y+A g^{k}(y)+\sum_{j=2}^{j_{m}} \tilde{X}^{j}\left(y+g^{k}(y)\right)\right)
$$

Since $g^{k}$ is a strictly non linear map in $H^{1, r}\left(\mathbb{R}^{n}\right)$, we have $\left(\partial g_{i}^{k} / \partial y_{s}\right)(y) \equiv$ 0 , for all $1 \leq i \leq s \leq n$ ( $g_{i}^{k}$ denoting the $i$-th components of the map $\left.g^{k}\right)$ which implies that the Jacobian $\left(\partial g^{k} / \partial y\right)(y)$ is a lower triangular matrix with zeros on the diagonal and hence a nilpotent matrix. Therefore we can write

$$
\left(I+\frac{\partial g^{k}}{\partial y}(y)\right)^{-1}=\sum_{s=0}^{N}(-1)^{s}\left(\frac{\partial g^{k}}{\partial y}(y)\right)^{s}
$$

for some $N \in \mathbb{N}$. Substitution of this into (3.6) and expansion of the resulting expression (using the analyticity of the fields $\tilde{X}^{j}$ ), retaining only 
homogeneous terms of degree $k$ (with respect to the standard dilation) and lower, produces

$$
A y+A g^{k}(y)+\sum_{j=2}^{k} \tilde{X}^{j}(y)-\left(\frac{\partial g^{k}}{\partial y}(y)\right) A y
$$

which can be rewritten in the form

$$
A y+\sum_{j=2}^{k} \tilde{X}^{j}(y)+\left[A, g^{k}\right](y)
$$

Observe now that $g^{k} \in H^{k, 1}\left(\mathbb{R}^{n}\right) \cap H^{1, r}\left(\mathbb{R}^{n}\right)$ and $A \in H^{1,1}\left(\mathbb{R}^{n}\right) \cap$ $H^{1, r}\left(\mathbb{R}^{n}\right)$, imply $\left[A, g^{k}\right] \in H^{k, 1}\left(\mathbb{R}^{n}\right) \cap H^{1, r}\left(\mathbb{R}^{n}\right)$. Thus the map

$$
g^{k} \longmapsto a d_{k} A\left(g^{k}\right)=\left[A, g^{k}\right]
$$

is a linear operator acting on the linear space $H^{k, 1}\left(\mathbb{R}^{n}\right) \cap H^{1, r}\left(\mathbb{R}^{n}\right)$.

At this point we need to state a technical lemma that is proved after the theorem using a standard argument in normal form theory (e.g., see [22, Coroll. 2.1] and [23, Thm. 2.5]).

Lemma 3.2. - Let $A$ be a linear map in $H^{1, r}\left(\mathbb{R}^{n}\right)$ and $S$ the semisimple part of $A$. Denote by $\operatorname{Ker}\left(a d_{k} S\right)$ and $\operatorname{Im}\left(a d_{k} A\right)$ respectively the kernel and the range of the linear operators $a d_{k} S, a d_{k} A$ defined as in (3.8), with $k \geq 1$. Then there exists a subspace $V_{k}$ of $K \operatorname{er}\left(a d_{k} S\right)$ that is a complement to $\operatorname{Im}\left(a d_{k} A\right)$ in $H^{k, 1}\left(\mathbb{R}^{n}\right) \cap H^{1, r}\left(\mathbb{R}^{n}\right)$, i.e. such that

$$
H^{k, 1}\left(\mathbb{R}^{n}\right) \cap H^{1, r}\left(\mathbb{R}^{n}\right)=\operatorname{Im}\left(a d_{k} A\right) \oplus V_{k}
$$

Using this lemma we can find $g^{k}, h^{k} \in H^{k, 1}\left(\mathbb{R}^{n}\right) \cap H^{1, r}\left(\mathbb{R}^{n}\right)$, such that

$$
-\tilde{X}^{k}=a d_{k} A\left(g^{k}\right)+h^{k}, \quad\left[S, h^{k}\right]=0 .
$$

Consequently, after the transformation (3.5) with $g^{k}$ chosen as in (3.10), the field (3.4) takes the form (3.3), which concludes the proof.

Proof of Lemma 3.2. - Denote by $\widehat{N}$ the nilpotent part of $A$. We first show that $a d_{k} A=a d_{k} S+a d_{k} \widehat{N}$ is the semisimple-nilpotent decomposition of the linear operator $a d_{k} A$. Since $[S, \widehat{N}]=0$ it follows, using the Jacobi identity, that $a d_{k} S$ and $a d_{k} \widehat{N}$ commute. To prove that $a d_{k} S$ is semisimple, by considering the natural isomorphism between the complexification $\left(H^{k, 1}\left(\mathbb{R}^{n}\right) \cap H^{1, r}\left(\mathbb{R}^{n}\right)\right)^{c}$ of the space $H^{k, 1}\left(\mathbb{R}^{n}\right) \cap H^{1, r}\left(\mathbb{R}^{n}\right)$, 
and the space $H^{k, 1}\left(\mathbb{C}^{n}\right) \cap H^{1, r}\left(\mathbb{C}^{n}\right)$ (which is defined in analogous way to the corresponding real one), it will be sufficient to show that $H^{k, 1}\left(\mathbb{C}^{n}\right) \cap H^{1, r}\left(\mathbb{C}^{n}\right)$ has a basis consisting of eigenvectors of $a d_{k} S^{c}$ (where $S^{c}$ denotes the complexification of the map $S$ ). Moreover, let $B \in H^{1,1}\left(\mathbb{C}^{n}\right) \cap H^{1, r}\left(\mathbb{C}^{n}\right)$ be the linear transformation that puts $S^{c}$ in diagonal form $T_{B} S^{c}=B^{-1} S^{c} B=D$. Then, by Theorem 2.1., $T_{B}$ is an invertible linear operator on $H^{k, 1}\left(\mathbb{C}^{n}\right) \cap H^{1, r}\left(\mathbb{C}^{n}\right)$. Hence, it will be equivalent to show that there exists a basis of $H^{k, 1}\left(\mathbb{C}^{n}\right) \cap H^{1, r}\left(\mathbb{C}^{n}\right)$ consisting of eigenvectors of $T_{B} \circ\left(a d_{k} S^{c}\right) \circ T_{B^{-1}}=a d_{k} T_{B} S^{c}=a d_{k} D$, where $D=\operatorname{diag}\left\{\lambda_{1}, \ldots \lambda_{n}\right\}$ is a diagonal linear map with $\lambda_{1}, \ldots \lambda_{n}$ denoting the eigenvalues of $S$ (and therefore of $A$ ). If we denote by $\left\{e_{1}, \ldots, e_{n}\right\}$ the canonical basis of $\mathbb{C}^{n}$, the set

$$
\mathcal{B}=\left\{x^{\nu} e_{i}: 1 \leq i \leq n, \nu=\left(\nu_{1}, \ldots \nu_{n}\right) \in \mathbb{N}^{n}, \sum_{s=1}^{n} \nu_{s}=k, \sum_{s=1}^{n} \nu_{s} r_{s}=r_{i}\right\}
$$

constitutes a basis for $H^{k, 1}\left(\mathbb{C}^{n}\right) \cap H^{1, r}\left(\mathbb{C}^{n}\right)$. A straightforward computation shows that, for any $x^{\nu} e_{i} \in \mathcal{B}$, we have

$$
a d_{k} D\left(x^{\nu} e_{i}\right)=\left(\lambda_{i}-\sum_{s=1}^{n} \nu_{s} \lambda_{s}\right) x^{\nu} e_{i}
$$

thus proving that the elements of $\mathcal{B}$ are eigenvectors of $\operatorname{ad}_{k} D$ with corresponding eigenvalues of the form

$$
\begin{gathered}
\mu_{\nu, i}=\lambda_{i}-\sum_{s=1}^{n} \nu_{s} \lambda_{s}, \quad 1 \leq i \leq n, \nu=\left(\nu_{1}, \ldots \nu_{n}\right) \in \mathbb{N}^{n}, \\
\sum_{s=1}^{n} \nu_{s}=k, \sum_{s=1}^{n} \nu_{s} r_{s}=r_{i} .
\end{gathered}
$$

Regarding the nilpotency of $a d_{k} \widehat{N}$, observe that for any $Z \in H^{k, 1}\left(\mathbb{R}^{n}\right) \cap$ $H^{1, r}\left(\mathbb{R}^{n}\right)$ and for any $j>k$, we have $D^{j} Z=0$ (denoting by $D^{j} Z$ the $j$-th order differential of $Z$ ). Then, since $\left(a d^{m} \widehat{N}, Z\right)(x)$ is a linear combination of elements of the form

$$
\widehat{N}^{s} D^{t} Z(x)\left[\widehat{N}^{i_{1}} x, \ldots, \widehat{N}^{i_{t}} x\right], \quad 0 \leq s, t \leq m, i_{t} \leq \ldots \leq i_{1},
$$

with $t=i_{1}=0$ when $s=m$, and $1 \leq i_{1}, t+i_{1}=m+1-s$ when $s<m$, it follows that $\left(a d_{k} \widehat{N}\right)^{3 p}=0$ for $p>\max \{k, \bar{n}\}$, where $\bar{n}$ denotes the nilpotency order of $\widehat{N}$. 
Thus $a d_{k} S$ constitutes the semisimple part in the Jordan decomposition of the operator $a d_{k} A$, which implies that the kernel of the adjoint operator $\left(a d_{k} A\right)^{T}$ is a subspace of the kernel of $a d_{k} S$. Moreover, by simple facts from linear algebra (the Fredholm alternative) we know that the kernel of $\left(a d_{k} A\right)^{T}$ is a complement space to $\operatorname{Im}\left(a d_{k} A\right)$. Thus $\operatorname{Ker}\left(\left(a d_{k} A\right)^{T}\right)$ is a possible choice for a subspace of $\operatorname{Ker}\left(a d_{k} S\right)$ that satisfies (3.9).

Remark 3.3. - Denote by $C\left(H^{1,1}\left(\mathbb{R}^{n}\right) \cap H^{1, r}\left(\mathbb{R}^{n}\right)\right)$ the center of the Lie algebra $H^{1,1}\left(\mathbb{R}^{n}\right) \cap H^{1, r}\left(\mathbb{R}^{n}\right)$. It is not difficult to verify that $\left(H^{1,1}\left(\mathbb{R}^{n}\right) \cap H^{1, r}\left(\mathbb{R}^{n}\right)\right) / C\left(H^{1,1}\left(\mathbb{R}^{n}\right) \cap H^{1, r}\left(\mathbb{R}^{n}\right)\right)$ is a finite dimensional semisimple Lie algebra. Therefore, since the map $A \mapsto a d_{k} A$ is a representation of $H^{1,1}\left(\mathbb{R}^{n}\right) \cap H^{1, r}\left(\mathbb{R}^{n}\right)$ in $H^{k, 1}\left(\mathbb{R}^{n}\right) \cap H^{1, r}\left(\mathbb{R}^{n}\right)$, the semisimple-nilpotent decomposition $a d_{k} A=a d_{k} S+a d_{k} \widehat{N}$ in the proof of lemma 3.2 can also be derived from a well known result in the theory of Lie algebras (see [15, Coroll. 6.4]).

Remark 3.4. - Adapting results analogous to lemma 3.2, of A. Vanderbauwhede [21], and C. Elphick et al. [7], it can be easily shown that we can introduce a particular inner product on $H^{k, 1}\left(\mathbb{R}^{n}\right) \cap H^{1, r}\left(\mathbb{R}^{n}\right)$ such that the adjoint operator satisfies $\left(a d_{k} A\right)^{T}=a d_{k} A^{T}$. Thus we may choose the kernel of $a d_{k} A^{T}$ as a subspace $V_{k}$ of $H^{k, 1}\left(\mathbb{R}^{n}\right) \cap H^{1, r}\left(\mathbb{R}^{n}\right)$ that satisfies the condition of the lemma.

Example 3.5. - (i) On $\mathbb{R}^{3}$ consider the field

$$
X(x)=x_{1} \frac{\partial}{\partial x_{1}}-x_{2} \frac{\partial}{\partial x_{2}}+\left(3 x_{3}+5 x_{1}^{3}-x_{1}^{2} x_{2}+2 x_{1} x_{2}^{2}+7 x_{2}^{3}\right) \frac{\partial}{\partial x_{3}} .
$$

Note that $X \in H^{1, r}\left(\mathbb{R}^{3}\right)$, with $r=(1,1,3)$, and that its linear part $A$ is represented by a symmetric matrix. Therefore, by the previous remark, we can choose a complementary space to $\operatorname{Im}\left(a d_{3} A\right)$ satisfying (3.9) to be the kernel of $a d_{3} A$, that is equal to $\operatorname{span}\left\{x_{1}^{3} \partial / \partial x_{3}\right\}$. In fact we have $\operatorname{Im}\left(a d_{3} A\right)=\operatorname{span}\left\{x_{1}^{2} x_{2} \partial / \partial x_{3}, x_{1} x_{2}^{2} \partial / \partial x_{3}, x_{2}^{3} \partial / \partial x_{3}\right\}$ and we may verify that, if we set

$$
g^{3}(x)=\left(\frac{1}{2} x_{1}^{2} x_{2}-\frac{1}{2} x_{1} x_{2}^{2}-\frac{7}{6} x_{2}^{3}\right) \frac{\partial}{\partial x_{3}}, \quad h^{3}(x)=5 x_{1}^{3} \frac{\partial}{\partial x_{3}},
$$

we can write $X=A-\left[A, g^{3}\right]+h^{3}, h^{3} \in \operatorname{Ker}\left(a d_{3} A\right)$. Thus, after performing the coordinate transformation

$$
x=\phi(y)=\left(\begin{array}{c}
y_{1} \\
y_{2} \\
y_{3}+(1 / 2) y_{1}^{2} y_{2}-(1 / 2) y_{1} y_{2}^{2}-(7 / 6) y_{2}^{3}
\end{array}\right)
$$


the field $X$ takes the desired form (1.3), (1.4):

$$
\begin{array}{r}
T_{\phi} X(y)=\left(y_{1} \frac{\partial}{\partial y_{1}}-y_{2} \frac{\partial}{\partial y_{2}}+3 y_{3} \frac{\partial}{\partial y_{3}}\right)+5 y_{1}^{3} \frac{\partial}{\partial y_{3}} \\
{\left[y_{1} \frac{\partial}{\partial y_{1}}-y_{2} \frac{\partial}{\partial y_{2}}+3 y_{3} \frac{\partial}{\partial y_{3}}, 5 y_{1}^{3} \frac{\partial}{\partial y_{3}}\right]=0}
\end{array}
$$

(ii) On $\mathbb{R}^{4}$ consider the field

$$
\begin{aligned}
X(x)=-x_{2} \frac{\partial}{\partial x_{1}}+x_{1} \frac{\partial}{\partial x_{2}} & +\left(-x_{4}+5 x_{1}^{3}-6 x_{1}^{2} x_{2}+5 x_{1} x_{2}^{2}+x_{2}^{3}\right) \frac{\partial}{\partial x_{3}} \\
& +\left(x_{3}-2 x_{1}^{3}+3 x_{1}^{2} x_{2}+3 x_{1} x_{2}^{2}+11 x_{2}^{3}\right) \frac{\partial}{\partial x_{4}}
\end{aligned}
$$

Note that $X \in H^{1, r}\left(\mathbb{R}^{4}\right)$, with $r=(1,1,3,3)$, and that its linear part $A$ is a semisimple field with double, purely imaginary eigenvalues $\lambda_{1,2}= \pm i$. An easy calculation shows that the kernel of $a d_{3} A^{T}$ is equal to $\operatorname{span}\left\{\left(x_{1}^{3}+\right.\right.$ $\left.\left.x_{1} x_{2}^{2}\right) \partial / \partial x_{3}+\left(x_{1}^{2} x_{2}+x_{2}^{3}\right) \partial / \partial x_{4},\left(x_{1}^{2} x_{2}+x_{2}^{3}\right) \partial / \partial x_{3}-\left(x_{1}^{3}+x_{1} x_{2}^{2}\right) \partial / \partial x_{4}\right\}$, and that the range of $a d_{3} A$ is equal to $\operatorname{span}\left\{3 x_{1}^{2} x_{2} \partial / \partial x_{3}+x_{1}^{3} \partial / \partial x_{4}\right.$, $\left(-x_{1}^{3}+x_{1} x_{2}^{2}\right) \partial / \partial x_{3}+x_{1}^{2} x_{2} \partial / \partial x_{4},\left(-2 x_{1}^{2} x_{2}+x_{2}^{3}\right) \partial / \partial x_{3}+x_{1} x_{2}^{2} \partial / \partial x_{4}$, $-3 x_{1} x_{2}^{2} \partial / \partial x_{3}+x_{2}^{3} \partial / \partial x_{4},-x_{1}^{3} \partial / \partial x_{3}+3 x_{1}^{2} x_{2} \partial / \partial x_{4},-x_{2}^{3} \partial / \partial x_{3}-$ $\left.3 x_{1} x_{2}^{2} \partial / \partial x_{4}\right\}$. Let $X^{3}=\left(5 x_{1}^{3}-6 x_{1}^{2} x^{2}+5 x_{1} x_{2}^{2}+x_{2}^{3}\right) \partial / \partial x_{3}+\left(-2 x_{1}^{3}+\right.$ $\left.3 x_{1}^{2} x_{2}+3 x_{1} x_{2}^{2}+11 x_{2}^{3}\right) \partial / \partial x_{4}$ be the homogeneous part of degree 3 (with respect to the standard dilation) of $X$. Then, by the previous remark, we can write $X^{3}=-\left[A, g^{3}\right]+h^{3}$, with $g^{3} \in H^{3,1}\left(\mathbb{R}^{4}\right) \cap H^{1, r}\left(\mathbb{R}^{4}\right)$, and $h^{3} \in \operatorname{Ker}\left(a d_{3} A^{T}\right)$. Indeed, this equality is satisfied with

$$
\begin{aligned}
& g^{3}(x)=\left(2 x_{1}^{3}-5 x_{1}^{2} x_{2}-4 x_{2}^{3}\right) \frac{\partial}{\partial x_{3}}+\left(3 x_{1}^{3}+x_{2}^{3}\right) \frac{\partial}{\partial x_{4}} \\
& h^{3}(x)=\left(7 x_{1}^{3}+7 x_{1} x_{2}^{2}\right) \frac{\partial}{\partial x_{3}}+\left(7 x_{1}^{2} x_{2}+7 x_{2}^{3}\right) \frac{\partial}{\partial x_{4}}
\end{aligned}
$$

Therefore, the coordinate transformation

$$
x=\phi(y)=\left(\begin{array}{c}
y_{1} \\
y_{2} \\
y_{3}+2 y_{1}^{3}-5 y_{1}^{2} y_{2}-4 y_{2}^{3} \\
y_{4}+3 y_{1}^{3}+y_{2}^{3}
\end{array}\right)
$$


gives to the field $X$ the form (1.3), (1.4):

$$
\begin{aligned}
& T_{\phi} X(y)=-y_{2} \frac{\partial}{\partial y_{1}}+y_{1} \frac{\partial}{\partial y_{2}}+\left(-y_{4}+7 y_{1}^{3}+7 y_{1} y_{2}^{2}\right) \frac{\partial}{\partial y_{3}} \\
& \left.+\left(y_{3}+7 y_{1}^{2} y_{2}+7 y_{2}^{3}\right)\right) \frac{\partial}{\partial y_{4}} \\
& {\left[-y_{2} \frac{\partial}{\partial y_{1}}+y_{1} \frac{\partial}{\partial y_{2}}-y_{4} \frac{\partial}{\partial y_{3}}+y_{3} \frac{\partial}{\partial y_{4}},\left(7 y_{1}^{3}+7 y_{1} y_{2}^{2}\right) \frac{\partial}{\partial y_{3}}\right.} \\
& \left.\quad+\left(7 y_{1}^{2} y_{2}+7 y_{2}^{3}\right) \frac{\partial}{\partial y_{4}}\right]=0 .
\end{aligned}
$$

Remark 3.6. - The constructive proof of Theorem 3.1 permits one to compute a normal form for $X$ satisfying (1.3), (1.4) in a finite number of steps $j \leq j_{m}$. Such a procedure cannot be applied to vector fields that are not homogeneous of degree one with respect to a dilation $\delta_{\varepsilon}^{r}$. In fact in this latter case, since we cannot use Theorem 2.1, any transformation $\phi_{k}$ of the form (3.5) introduces additional higher order terms that are no more necessarily homogeneous of degree one with respect to the dilation $\delta_{\varepsilon}^{r}$. Thus we would produce an infinite sequence $\left\{\phi_{k}\right\}_{k}, k \in \mathbb{N}$, and it would be necessary to study the convergence to a function of the corresponding products $\left\{\phi_{1} \circ \ldots \circ \phi_{k}\right\}_{k}$.

Remark 3.7. - Theorem 3.1 can also be derived from a result of K.T. Chen [6, Thm. 8.1] who proved the existence of a formal transformation (i.e. a transformation given by a formal series) that puts a formal vector field of a graded Lie algebra into the form (1.3), (1.4). Our approach is a constructive treatment of the formal result of Chen and yields an explicit procedure to compute a polynomial coordinate change that gives to a field $X \in H^{1, r}\left(\mathbb{R}^{n}\right)$ the normal form (1.3), (1.4).

Remark 3.8. - The decomposition (1.3), (1.4) of a field $X \in H^{1, r}\left(\mathbb{R}^{n}\right)$ produces a normal form for all the elements of the group orbit $T_{\phi} X$, $\phi \in G H^{1, r}\left(\mathbb{R}^{n}\right)$. This normal form is not unique. Indeed its linear part can be uniquely determined if we require it to be in Jordan canonical form (or in real canonical form in the case it has some non real eigenvalue) which can be done by performing, after obtaining (1.3), a further coordinate linear transformation $y=\psi(z), \psi \in G H^{1, r}\left(\mathbb{R}^{n}\right)$, that clearly preserves (1.4). However, the choice of the complementary spaces $V_{k}$ to $\operatorname{Im}\left(a d_{k} A\right)$ that satisfy condition (3.9) of the lemma, is in general far from being unique and therefore some arbitrariness in the form of the non linear part is unavoidable. 


\subsection{An application}

We present here a corollary of Theorem 3.1 that provides a Poincaré type result for homogeneous vector fields of degree one with respect to a dilation $\delta_{\varepsilon}^{r}, r=\left(r_{1}, \ldots, r_{n}\right)$ of the type $\left(i_{1}, \ldots, i_{m} ; j_{1}, \ldots, j_{m}\right)$ as defined in (2.1). In fact a well known theorem proved by Poincaré shows that a sufficient condition for the existence of a (formal) coordinate change that takes a given vector field $X$ into a linear field is that the eigenvalues $\lambda_{1}, \ldots, \lambda_{n}$ of the linear part of $X$ do not satisfy any resonance relation of order $k \geq 2$, i.e. any relation of the form

$$
\lambda_{i}=\sum_{s=1}^{n} \nu_{s} \lambda_{s}, \quad \nu_{s} \in \mathbb{N}, \quad \sum_{s=1}^{n} \nu_{s}=k
$$

For vector fields $X \in H^{1, r}\left(\mathbb{R}^{n}\right)$ it turns out to be sufficient to check that the eigenvalues do not satisfy only a finite number of renonance relations.

COROLlaRY 3.9. - Let $X$ be a real analytic vector field in $H^{1, r}\left(\mathbb{R}^{n}\right)$. Denote by

$$
\lambda_{1}=\lambda_{i_{0}}, \ldots, \lambda_{i_{1}}, \lambda_{\left(i_{1}+1\right)}, \ldots, \lambda_{i_{2}}, \ldots, \lambda_{\left(i_{m-1}+1\right)}, \ldots, \lambda_{i_{m}}=\lambda_{n}
$$

the eigenvalues of the linear part $A=\operatorname{diag}\left\{A_{1}, \ldots, A_{m}\right\}$ of $X$ ordered so that $\lambda_{\left(i_{j-1}+1\right)}, \ldots, \lambda_{i_{j}}, j=1, \ldots, m$, are the eigenvalues of the $j$-th block $A_{j}$ of $A$. Suppose that $\lambda_{1}, \ldots, \lambda_{n}$ do not satisfy any relation of the form

$$
\lambda_{i}=\sum_{s=1}^{n} \nu_{s} \lambda_{s}
$$

with $\nu_{s} \in \mathbb{N}$ such that

$$
r_{i}=\sum_{s=1}^{n} \nu_{s} r_{s}, \quad \sum_{s=1}^{n} \nu_{s}=k, \quad \text { for some } k\left(2 \leq k \leq j_{m}\right) .
$$

Then there exists a polynomial change of coordinates $\phi \in G H^{1, r}\left(\mathbb{R}^{n}\right)$ such that

$$
T_{\phi} X=A
$$

$A$ relation among the eigenvalues of the form (3.14), with $\nu_{s} \in \mathbb{N}$ satisfying (3.15), will be called a resonance with respect to the dilation $\delta_{\varepsilon}^{r}$. 
Proof. - The proof of lemma 3.2 shows that the semisimple part of each operator $a d_{k} A$ is given by the operator $a d_{k} S$ (with $S$ being the semisimple part of $A$ ) and that the eigenvalues of $a d_{k} S$ have the form (3.13) $\mu=\lambda_{i}-\sum_{s=1}^{n} \nu_{s} \lambda_{s}$, with $\sum_{s=1}^{n} \nu_{s} r_{s}=r_{i}, \sum_{s=1}^{n} \nu_{s}=k$. Since the non resonance relations with respect to $\delta_{\varepsilon}^{r}$ of the eigenvalues $\lambda_{1}, \ldots, \lambda_{n}$ imply that the eigenvalues of $a d_{k} S$ (and therefore of $a d_{k} A$ ) are all different from zero, the operators $a d_{k} A$ are invertible for $2 \leq k \leq j_{m}$. Hence the transformation $\phi=\phi_{2} \circ \ldots \circ \phi_{j_{m}}$ constructed in Theorem 3.1 gives $X$ the form (3.16).

Example 3.10. - (i) On $\mathbb{R}^{3}$ consider the vector field

$$
X(x)=\left(2 x_{1}+x_{2}\right) \frac{\partial}{\partial x_{1}}+2 x_{2} \frac{\partial}{\partial x_{2}}+\left(x_{1}^{2}+2 x_{1} x_{2}-x_{2}^{2}\right) \frac{\partial}{\partial x_{3}} .
$$

Observe that $X \in H^{1, r}\left(\mathbb{R}^{n}\right)$, with $r=(1,1,2)$. The eigenvalues of the linear part of $X$ are $\lambda_{1}=\lambda_{2}=2, \lambda_{3}=0$. Note that there is no resonance of order 2 with respect to $\delta_{\varepsilon}^{r}$ :

$$
\lambda_{3} \neq 2 \lambda_{1}, \quad \lambda_{3} \neq \lambda_{1}+\lambda_{2}, \quad \lambda_{3} \neq 2 \lambda_{2} .
$$

Therefore, by Corollary 3.9, we can find a coordinate change $\phi \in$ $G H^{1, r}\left(\mathbb{R}^{n}\right)$ which transforms the field $X$ into its linear part. In fact, if we set

$$
x=\phi(y)=\left(\begin{array}{c}
y_{1} \\
y_{2} \\
y_{3}+(1 / 4) y_{1}^{2}+(3 / 80) y_{1} y_{2}-(11 / 32) y_{2}^{2}
\end{array}\right),
$$

we obtain

$$
T_{\phi} X(y)=\left(2 y_{1}+y_{2}\right) \frac{\partial}{\partial y_{1}}+2 y_{2} \frac{\partial}{\partial y_{2}} .
$$

It may be noted that it was possible to transform the field $X$ into its linear part even if there were resonances of any order of the form (3.14) not satisfying (3.15) (i.e. resonances in the classical sense) due to the eigenvalue $\lambda_{3}=0$ and in particular there were the following resonances of order 2 :

$$
\lambda_{1}=\lambda_{2}+\lambda_{3}, \quad \lambda_{2}=\lambda_{1}+\lambda_{3} .
$$

(ii) On $\mathbb{R}^{2}$ consider the vector field

$$
X(x)=\left(x_{1}+x_{2}^{2}\right) \frac{\partial}{\partial x_{1}}+\left(-x_{2}+x_{1}^{2}\right) \frac{\partial}{\partial x_{2}} .
$$


The eigenvalues of the linear part are $\lambda_{1}=1, \lambda_{2}=-1$. Observe that there is no classical resonance of order 2 since the eigenvalues do not satisfy any relation of the form (3.14) with $\nu_{1}+\nu_{2}=2$ :

$$
\lambda_{1}, \lambda_{2} \neq 0, \quad \lambda_{1} \neq 2 \lambda_{2}, \quad \lambda_{2} \neq 2 \lambda_{1}
$$

Thus we can find a coordinate transformation $\phi$ which removes completely the quadratic terms. If we set

$$
x=\phi(y)=\left(\begin{array}{l}
y_{1}-(1 / 3) y_{2}^{2} \\
y_{2}+(1 / 3) y_{1}^{2}
\end{array}\right)
$$

we obtain

$$
\begin{aligned}
T_{\phi} X(y)=\left(y_{1}+\frac{2}{3} y_{1}^{2} y_{2}+o\left(\left|y_{1}\right|^{3},\left|y_{2}\right|^{3}\right)\right) & \frac{\partial}{\partial y_{1}} \\
& +\left(-y_{2}-\frac{2}{3} y_{1} y_{2}^{2}+o\left(\left|y_{1}\right|^{3},\left|y_{2}\right|^{3}\right)\right) \frac{\partial}{\partial y_{2}} .
\end{aligned}
$$

Note that $(2 / 3) y_{1}^{2} y_{2} \partial / \partial y_{1},-(2 / 3) y_{1} y_{2}^{2} \partial / \partial y_{2}$ are resonant terms of order 3 in the classical sense: the eigenvalues satisfy the following relations of the form (3.14)

$$
\lambda_{1}=2 \lambda_{1}+\lambda_{2}, \quad \lambda_{2}=\lambda_{1}+2 \lambda_{2} .
$$

Thus these terms cannot be removed by further coordinate transformations. In this case the field $X$ is not homogeneous of degree one with respect to any dilation. Therefore the lack of classical resonances of order 2 is not sufficient to guarantee the existence of a coordinate change that transforms $X$ into its linear part.

\section{REPRESENTATION OF SOLUTIONS FOR A CLASS OF NONLINEAR SYSTEMS}

In this section we first derive a general representation formula for the solutions of an autonomous system of differential equations

$$
\dot{x}=X(x), \quad X \in H^{1, r}\left(\mathbb{R}^{n}\right) .
$$


Then we use this result to obtain a representation for the trajectories of an $n$-dimensional, single input, affine control system

$$
\begin{aligned}
& \dot{x}=X_{0}(x)+B u, \quad X_{0} \in H^{1, r}\left(\mathbb{R}^{n}\right), \\
& B=\left(\begin{array}{c}
b_{1} \\
\vdots \\
b_{n}
\end{array}\right) \in \mathbb{R}^{n}, \quad b_{i}=0, i=1, \ldots, i_{m-1},
\end{aligned}
$$

$\left(i_{m-1}+1\right.$ denoting as in (2.1) the smallest index $i$ such that $\left.r_{i}=r_{n}\right)$ in terms of integrals of the control $u$, which is analogous to the standard representation of the trajectories of a linear control system $\dot{x}=A x+b u$, ( $A, b$, denoting $n \times n, n \times 1$ matrices). In fact, a system of the form (4.2) coincides with a linear system when $\delta_{\varepsilon}^{r}$ is the standard dilation $\delta_{\varepsilon}^{1}$ with $r_{1}=\ldots=r_{n}=1$. Thus, systems of the form (4.2) can be regarded as a natural extension of the linear control systems.

\subsection{Representation of the flow generated by a homogeneous field of degree one with respect to an arbitrary dilation}

THEOREM 4.1. - Let $N$ be a vector field in $H^{1, r}\left(\mathbb{R}^{n}\right)$ of the form

$$
N=\widehat{N}+Y, \quad \widehat{N}, Y \in H^{1, r}\left(\mathbb{R}^{n}\right),
$$

where $\widehat{N}$ is a linear nilpotent field and $Y$ is a strictly non linear field. If $\phi_{k}(t, p), k \in \mathbb{N}$, denotes the Picard approximations of the solution to $\dot{x}=N(x), x(0)=p$, recursively defined by

$$
\begin{aligned}
\phi_{0}(t, p) & =p \\
\phi_{k+1}(t, p) & =p+\int_{0}^{t} N\left(\phi_{k}(s, p)\right) d s, \quad k=0,1, \ldots,
\end{aligned}
$$

then there exists an integer $\bar{k}$ such that

$$
(\exp t N)(p)=\phi_{\bar{k}}(t, p), \quad t \in \mathbb{R}, \quad p \in \mathbb{R}^{n}
$$

Proof. - Denote by $\phi_{k, i}$ the $i$-th component of the $k$-th Picard approximation $\phi_{k}$. We will show, by induction on the index $i, 1 \leq i \leq n$, that there exists a non decreasing $n$-tuple of positive integers $k_{1} \leq k_{2} \leq \ldots \leq k_{n}$, such that

$$
\phi_{k, i}(t, p)=\phi_{k+1, i}(t, p), \quad t \in \mathbb{R}, \quad p \in \mathbb{R}^{n}, \quad \forall k \geq k_{i} .
$$


This implies that, if we set $\bar{k}=k_{n}$, we have

$$
(\exp t N)(p)=\lim _{k \rightarrow \infty} \phi_{k}(t, p)=\phi_{\bar{k}}(t, p), \quad t \in \mathbb{R}, \quad p \in \mathbb{R}^{n},
$$

which is what we need to prove.

Since $\widehat{N}$ is a linear nilpotent element of $H^{1, r}\left(\mathbb{R}^{n}\right)$, it follows that (with the usual notation of Section 2) $\widehat{N}=\operatorname{diag}\left\{\widehat{N}_{1}, \ldots \widehat{N}_{m}\right\}$, where each $\widehat{N}_{j}$ is a $\left(i_{j}-i_{j-1}\right) \times\left(i_{j}-i_{j-1}\right)$ nilpotent block. Denote by $n_{j}, j=1, \ldots, m$, the nilpotency order of $\widehat{N}_{j}$, i.e. the smallest positive integer $n$ such that $\widehat{N}_{j}^{n}=0$. Note that, by Theorem $2.5, \phi_{k}$ are homogeneous functions of degree one with respect to $\delta_{\varepsilon}^{r}$, in the $p$-variable. From the definition of $\phi_{k}$ and the general form of an element of $H^{1, r}\left(\mathbb{R}^{n}\right)$, it follows that, if we set $k_{1}=k_{2}=\ldots=k_{i_{1}}=n_{1}$, we have

$$
\begin{aligned}
& \left(\begin{array}{c}
\phi_{k, 1}(t, p) \\
\vdots \\
\phi_{k, i_{1}}(t, p)
\end{array}\right)=\sum_{s=0}^{k} \frac{t^{s}}{s !} \widehat{N}_{1}^{s}\left(\begin{array}{c}
p_{1} \\
\vdots \\
p_{i_{1}}
\end{array}\right)=\sum_{s=0}^{k+1} \frac{t^{s}}{s !} \widehat{N}_{1}^{s}\left(\begin{array}{c}
p_{1} \\
\vdots \\
p_{i_{1}}
\end{array}\right) \\
& =\left(\begin{array}{c}
\phi_{k+1,1}(t, p) \\
\vdots \\
\phi_{k+1, i_{1}}(t, p)
\end{array}\right), \quad t \in \mathbb{R}, \quad p \in \mathbb{R}^{n}, \quad \forall k \geq k_{i}, \quad i=1, \ldots, i_{1} .
\end{aligned}
$$

Suppose now that we have obtained $k_{1} \leq k_{2} \leq \ldots \leq k_{i_{j}}, 1 \leq j<m$, such that relation (4.5) is satisfied for $i=1, \ldots, i_{j}$. Then

$$
\phi_{k, i}(t, p)=\phi_{k+1, i}(t, p), \quad t \in \mathbb{R}, \quad p \in \mathbb{R}^{n}, \quad i=1, \ldots i_{j}, \quad k \geq k_{i_{j}} .
$$

Moreover, it can be easily verified that, for any integer $k>1$, the following equality holds

$$
\begin{array}{r}
\left(\begin{array}{c}
\phi_{k, i_{j}+1}(t, p) \\
\vdots \\
\phi_{k, i_{(j+1)}}(t, p)
\end{array}\right)=\sum_{s=0}^{k} \frac{t^{s}}{s !} \widehat{N}_{j+1}^{s}\left(\begin{array}{c}
p_{i_{j}+1} \\
\vdots \\
p_{i_{(j+1)}}
\end{array}\right)+\frac{t^{k}}{k !} \widehat{N}_{j+1}^{k-1}\left(\begin{array}{c}
Y_{i_{j}+1}(p) \\
\vdots \\
Y_{i_{(j+1)}}(p)
\end{array}\right) \\
+\int_{0}^{t} \int_{0}^{t_{k-2}} \int_{0}^{t_{k-3}} \cdots \int_{0}^{t_{1}} \widehat{N}_{j+1}^{k-2}\left(\begin{array}{c}
Y_{i_{j}+1}\left(\phi_{1}(v, p)\right) \\
\vdots \\
Y_{i_{(j+1)}}\left(\phi_{1}(v, p)\right)
\end{array}\right) d v d t_{1} \ldots d t_{k-2} \\
+\ldots+\int_{0}^{t}\left(\begin{array}{c}
Y_{i_{j}+1}\left(\phi_{k-1}(v, p)\right) \\
\vdots \\
\left.Y_{i_{(j+1)}\left(\phi_{k-1}(v, p)\right)}\right)
\end{array}\right) d v
\end{array}
$$


Hence, using relation (4.5) and nilpotency of $\widehat{N}_{j+1}$, it follows that, if we set $k_{i_{j}+1}=k_{i_{j}+2}=\ldots=k_{i_{(j+1)}}=k_{i_{j}}+n_{j+1}$, we have

$$
\begin{aligned}
& \left(\begin{array}{c}
\phi_{k, i_{j}+1}(t, p) \\
\vdots \\
\phi_{k, i_{\langle j+1)}}(t, p)
\end{array}\right)=\sum_{s=0}^{n_{(j+1)}-1} \frac{t^{s}}{s !} \widehat{N}_{j+1}^{s}\left(\begin{array}{c}
p_{i_{j}+1} \\
\vdots \\
p_{i_{(j+1)}}
\end{array}\right) \\
& +\int_{0}^{t} \int_{0}^{t_{n_{1}(j+1)}-1} \cdots \int_{0}^{t_{1}} \widehat{N}_{j+1}^{n_{(j+1)}-1}\left(\begin{array}{c}
Y_{i_{j}+1}\left(\phi_{k_{i_{j}}}(v, p)\right) \\
\vdots \\
Y_{i_{(j+1)}}\left(\phi_{k_{i_{j}}}(v, p)\right)
\end{array}\right) d v d t_{1} \ldots d t_{n_{(j+1)}-1} \\
& +\ldots+\int_{0}^{t}\left(\begin{array}{c}
Y_{i_{j}+1}\left(\phi_{k_{i_{j}}}(v, p)\right) \\
\vdots \\
Y_{i_{(j+1)}}\left(\phi_{k_{i_{j}}}(v, p)\right)
\end{array}\right) d v, \quad \forall k \geq k_{i_{(j+1)}}
\end{aligned}
$$

which proves the inductive step.

Remark 4.2. - It is clear, from the proof of Theorem 4.1, that the smallest integer $\bar{k}$ such that (4.4) is satisfied, is given by $\bar{k}=\sum_{j=1}^{m} n_{j}$, where $n_{j}$ denote the nilpotency orders of the blocks $\widehat{N}_{j}$ of the linear part of the field $N$. Thus the solution to the Cauchy problem $\dot{x}=N(x), x(0)=p$, with $N=\widehat{N}+Y \in H^{1, r}\left(\mathbb{R}^{n}\right)$ as in Theorem 4.1 , can be computed using the following recursive formula

$$
\begin{aligned}
& \left(\begin{array}{c}
(\exp t N)(p))_{1} \\
\vdots \\
(\exp t N)(p))_{i_{1}}
\end{array}\right)=\sum_{s=0}^{n_{1}-1} \frac{t^{s}}{s !} \widehat{N}_{1}^{s}\left(\begin{array}{c}
p_{1} \\
\vdots \\
p_{i_{1}}
\end{array}\right), \\
& \left(\begin{array}{c}
((\exp t N)(p))_{i_{j}+1} \\
\vdots \\
((\exp t N)(p))_{i_{(j+1)}}
\end{array}\right)=\sum_{s=0}^{n_{(j+1)}-1} \frac{t^{s}}{s !} \widehat{N}_{j+1}^{s}\left(\begin{array}{c}
p_{i_{j}+1} \\
\vdots \\
p_{i_{(j+1)}}
\end{array}\right) \\
& +\int_{0}^{t} \int_{0}^{t_{n}(j+1)^{-1}} \ldots \\
& \ldots \int_{0}^{t_{1}} \widehat{N}_{j+1}^{n_{(j+1)}-1}\left(\begin{array}{c}
Y_{i_{j}+1}((\exp v N)(p)) \\
\vdots \\
Y_{i_{(j+1)}}((\exp v N)(p))
\end{array}\right) d v d t_{1} \ldots d t_{n_{(j+1)}-1} \\
& +\ldots+\int_{0}^{t}\left(\begin{array}{c}
Y_{i_{j}+1}((\exp v N)(p)) \\
Y_{i_{(j+1)}}((\exp v N)(p))
\end{array}\right) d v, j=1, \ldots, m-1 .
\end{aligned}
$$


THEOREM 4.3. - Let $X$ be a real analytic vector field in $H^{1, r}\left(\mathbb{R}^{n}\right)$, and $\phi \in G H^{1, r}\left(\mathbb{R}^{n}\right)$ a change of coordinates that transforms $X$ into the canonical form $T_{\phi} X=S+N$ of Theorem 3.1. Then

$$
(\exp t X)(p)=\phi\left(e^{t S}(\exp t N)\left(\phi^{-1}(p)\right)\right), \quad t \in \mathbb{R}, \quad p \in \mathbb{R}^{n}
$$

where the flow $(\exp t N)\left(\phi^{-1}(p)\right)$ can be computed by the recursive formula (4.7).

Proof. - Note that

$$
(\exp t X)(p)=\phi\left(\left(\exp t T_{\phi} X\right)\left(\phi^{-1}(p)\right)\right)
$$

Since $[S, N]=0$ we can write

$$
\left(\exp t T_{\phi} X\right)(q)=(\exp t(S+N))(q)=(\exp t S) \circ(\exp t N)(q),
$$

which evaluated at $q=\phi^{-1}(p)$, gives (4.8) after applying $\phi$ and using (4.9).

Remark 4.4. - It may be noted that the formula (4.8) in Theorem 4.4, is analogous to the "generalized variation of constants formula", obtained by A.A Agrachev, R.V. Gamkrelidze and A.V. Sarychev in [1], that express the flow generated by a vector field $X_{\tau}+Y_{\tau}$ as a perturbation of the flow generated by $X_{\tau}$.

Example 4.5. - Consider the Cauchy problem on $\mathbb{R}^{4}$

$$
\dot{x}=X(x), \quad x(0)=p=\left(p_{1}, p_{2}, p_{3}, p_{4}\right),
$$

where $X \in H^{1, r}\left(\mathbb{R}^{4}\right), r=(1,1,2,2)$, is the vector field

$$
\begin{aligned}
X(x)=\left(2 x_{1}+x_{2}\right) \frac{\partial}{\partial x_{1}}+2 x_{2} \frac{\partial}{\partial x_{2}}+\left(4 x_{3}+\right. & \left.5 x_{1}^{2}-x_{1} x_{2}+x_{2}^{2}\right) \frac{\partial}{\partial x_{3}} \\
& +\left(-x_{4}+x_{1} x_{2}-3 x_{2}^{2}\right) \frac{\partial}{\partial x_{4}} .
\end{aligned}
$$

First we compute the coordinate transformation that puts $X$ into the canonical form $(1.3),(1.4)$. If $A$ denotes the linear part of $X$, and $S$ the semisimple part of $A$, it can be easily seen that $\left(5 x_{1}^{2}-x_{1} x_{2}+x_{2}^{2}\right) \partial / \partial x_{3} \in$ $\operatorname{Ker}\left(a d_{2} S\right)$, and that $\operatorname{span}\left\{x_{1} x_{2} \partial / \partial x_{4}, x_{2}^{2} \partial / \partial x_{4}\right\}$ is a subspace of $\operatorname{Im}\left(a d_{2} A\right)$. Indeed we may check that, if we set $g^{2}(x)=\left((1 / 5) x_{1} x_{2}-\right.$ $\left.(16 / 25) x_{2}^{2}\right) \partial / \partial x_{4}, \quad h^{2}(x)=\left(5 x_{1}^{2}-x_{1} x_{2}+x_{2}^{2}\right) \partial / \partial x_{3}$, we have 
$X=A-\left[A, g^{2}\right]+h^{2}$. Thus, the coordinate change $x=\phi(y), \phi=I+g^{2}$, transforms $X$ into the canonical form

$$
T_{\phi} X=S+N, \quad N(y)=y_{2} \frac{\partial}{\partial y_{1}}+\left(5 y_{1}^{2}-y_{1} y_{2}+y_{2}^{2}\right) \frac{\partial}{\partial y_{3}}
$$

Next, using formula (4.7) with $q=\left(q_{1}, q_{2}, q_{3}, q_{4}\right)$, we compute the flow generated by $N$

$$
\begin{aligned}
& \left(\begin{array}{l}
((\exp t N)(q))_{1} \\
((\exp t N)(q))_{2}
\end{array}\right)=\left(\begin{array}{l}
q_{1} \\
q_{2}
\end{array}\right)+t\left(\begin{array}{c}
q_{2} \\
0
\end{array}\right) \\
& \left(\begin{array}{c}
((\exp t N)(q))_{3} \\
((\exp t N)(q))_{4}
\end{array}\right)=\left(\begin{array}{l}
q_{3} \\
q_{4}
\end{array}\right)+\int_{0}^{t}\left(\begin{array}{c}
5\left(q_{1}+v q_{2}\right)^{2}-\left(q_{1}+v q_{2}\right) q_{2}+q_{2}^{2} \\
0
\end{array}\right) d v \\
& =\left(\begin{array}{c}
q_{3}+\left(5 q_{1}^{2}-q_{1} q_{2}+q_{2}^{2}\right) t+\left(5 q_{1} q_{2}-(1 / 2) q_{2}^{2}\right) t^{2}+(5 / 3) q_{2}^{2} t^{3} \\
q_{4}
\end{array}\right) .
\end{aligned}
$$

Then, multiplying the exponential matrix $e^{t S}$ by the above expression, after substituting in it $q=\phi^{-1}(p)=p-g^{2}(p)$, we obtain

$$
\begin{aligned}
& \left(\exp t T_{\phi} X\right)\left(\phi^{-1}(p)\right)= \\
& e^{2 t}\left(p_{1}+t p_{2}\right) \\
& e^{2 t} p_{2} \\
& \left(\begin{array}{c}
4 t \\
e^{4}\left(p_{3}+\left(5 p_{1}^{2}-p_{1} p_{2}+p_{2}^{2}\right) t+\left(5 p_{1} p_{2}-(1 / 2) p_{2}^{2}\right) t^{2}+(5 / 3) p_{2}^{2} t^{3}\right) \\
e^{-t}\left(p_{4}-(1 / 5) p_{1} p_{2}+(16 / 25) p_{2}^{2}\right)
\end{array}\right)
\end{aligned}
$$

which gives

$$
\begin{aligned}
& (\exp t X)(p)= \\
& \left(\begin{array}{c}
e^{2 t}\left(p_{1}+t p_{2}\right) \\
e^{2 t} p_{2} \\
e^{4 t}\left(p_{3}+\left(5 p_{1}^{2}-p_{1} p_{2}+p_{2}^{2}\right) t+\left(5 p_{1} p_{2}-(1 / 2) p_{2}^{2}\right) t^{2}+(5 / 3) p_{2}^{2} t^{3}\right) \\
e^{-t}\left(p_{4}-(1 / 5) p_{1} p_{2}+(16 / 25) p_{2}^{2}\right) \\
+e^{4 t}\left(\left((1 / 5) p_{1} p_{2}-(16 / 25) p_{2}^{2}\right)+(1 / 5) p_{2}^{2} t\right)
\end{array}\right)
\end{aligned}
$$

\subsection{Representation of the trajectories for a class of nonlinear control systems.}

Theorem 4.6. - Let $\dot{x}=X_{0}(x)+B u$, be an n-dimensional, single input, affine control system as in (4.2). Then, if we denote by $A$ the linear part of the field $X_{0}$, the trajectories $x(\cdot, u)$ of such a system can be computed 
with the formula

$$
x(t, u)=\left(\exp t X_{0}\right)\left(x(0)+\int_{0}^{t} e^{-s A} B u(s) d s\right), \quad t \in \mathbb{R} .
$$

Proof. - A direct calculation verifies that in order to show that (4.10) represents a trajectory of (4.2) we only need to prove

$$
\left(\left.\frac{\partial\left(\exp t X_{0}\right)}{\partial p}(p)\right|_{p=x(0)+\int_{0}^{t} e^{-s A} B u(s) d s}\right) e^{-t A} B=B, \quad t \in \mathbb{R} .
$$

Since $X_{0}$ is homogeneous of degree one with respect to $\delta_{\varepsilon}^{r}$, its linear part is represented by a diagonal matrix composed of blocks of order $\left(i_{k}-i_{k-1}\right) \times\left(i_{k}-i_{k-1}\right), k=1, \ldots, m$ (with notation of Section 2) and therefore also the matrix representing $e^{-t A}$ has the same form. It follows that $e^{-t A} B$ is a column vector having all components zero but the last $n-i_{m-1}$ like $B$. Hence in order to prove (4.11) it is sufficient to show

$$
\left(\frac{\partial\left(\exp t X_{0}\right)}{\partial p}(p)\right) C=e^{t A} C, \quad p \in \mathbb{R}^{n}, \quad t \in \mathbb{R}
$$

for any column vector $C$ having all components zero but the last $n-i_{m-1}$. It is a well-known fact, in the theory of differential equations, that the Jacobian $\left(\partial\left(\exp t X_{0}\right) / \partial p\right)(p)$ is equal to the fundamental matrix solution of the variational equation

$$
\dot{v}=\left(\left.\frac{\partial X_{0}}{\partial x}(x)\right|_{x=\left(\exp t X_{0}\right)(p)}\right) v=A v+\left(\left.\frac{\partial Y}{\partial x}(x)\right|_{x=\left(\exp t X_{0}\right)(p)}\right) v
$$

where $Y$ denotes the nonlinear part of $X_{0}$. Let $\tilde{v}_{j}, j=1, \ldots, n-i_{m-1}$ be the solutions of $\dot{\tilde{v}}=A_{m} \tilde{v}, \tilde{v}(0)=\tilde{e}_{j}$, where $\left\{\tilde{e}_{1}, \ldots \tilde{e}_{n-i_{m-1}}\right\}$ denotes the canonical basis of $\mathbb{R}^{n-i}$ and $A_{m}$ is the last block of the matrix $A$. Then define the functions $v_{j}, j=1, \ldots, n-i_{m-1}$ having zero the first $i_{m-1}$ components and equal to the ones of $\tilde{v}_{j}$ the last $n-i_{m_{-1}}$ components. It can be easily seen that $v_{j}$ are solutions of $\dot{v}=A v, v(0)=e_{j+i_{m-1}}$, ( $e_{j}$ being the canonical basis of $\mathbb{R}^{n}$ ) and therefore they constitute the last $n-i_{m-1}$ columns of the matrix $e^{t A}$. Observe now that the last $n-i_{m-1}$ columns of the Jacobian $(\partial Y / \partial x)(x)$ are all zero since from the definition of homogeneity of degree one it follows that any component of the non linear part $Y$ of $X_{0}$ is independent on the last $n-i_{m-1}$ variables. Therefore it can be easily verified that $v_{j}$ are also solutions of 4.13 and hence they 
constitute the last $n-i_{m-1}$ columns of $\left(\partial\left(\exp t X_{0}\right) / \partial p\right)(p)$ for any $p \in \mathbb{R}^{n}$. This shows that the last columns of the matrices $e^{t A},\left(\partial\left(\exp t X_{0}\right) / \partial p\right)(p)$ are the same, for any $p \in \mathbb{R}^{n}$, which proves (4.12).

Remark 4.7. - Observe that the first $i_{m-1}$ components of a map $\psi \in G H^{1, r}\left(\mathbb{R}^{n}\right)$ are independent on the last $n-i_{m-1}$ variables, while the last $n-i_{m-1}$ components depend linearly on the last $n-i_{m-1}$ variables. Therefore, if the first $i_{m-1}$ components of a column vector $B$ are zero and the last $n-i_{m-1}$ are constant, the same is true for the components of the vector $(\partial \psi / \partial y) B$. Thus it can be easily verified, using also Theorem 2.1 of Section 2, that any coordinate transformation $\phi \in G H^{1, r}\left(\mathbb{R}^{n}\right)$ transforms an affine system as (4.2) into a system of the same form. Hence we can always perform a coordinate change $x=\phi(y), \phi \in G H^{1, r}\left(\mathbb{R}^{n}\right)$ that transforms the field $X_{0}$ in (4.2) into the canonical form $T_{\phi} X_{0}=S+N,[S, N]=0$ of Theorem 3.1 and use the formula given in Theorem 4.3 to derive the following explicit representation of the trajectories of (4.2)

$$
\begin{gathered}
x(t, u)=\phi\left(e^{t S}(\exp t N)\left(\phi^{-1}\left(x(0)+\int_{0}^{t} e^{-s(S+\widehat{N})} B u(s) d s\right)\right)\right) \\
t \in \mathbb{R},
\end{gathered}
$$

where $\widehat{N}$ denotes the linear part of the field $N$.

Remark 4.8. - The flow generated by a homogeneous vector field of degree one can be in general calculated by solving a cascade system of the type

$$
\dot{x}_{i}=A_{i} x_{i}+Y_{i}\left(x_{1}, \ldots, x_{i-1}\right), \quad x_{i} \in \mathbb{R}^{d_{i}}, \quad d_{i} \in \mathbb{N} \backslash\{0\} .
$$

This implies that systems of the form (4.2) can be always integrated without any restriction on the constant vector field $B$. However, formula (4.10) allows to derive a general description of the attainable set of a nonlinear system of type (4.2) as the image, through the flow generated by $X_{0}$, of the attainable set of its linearized system.

Remark 4.9. - The representation of the trajectories of control systems of the form (4.2) given by formulas (4.10), (4.14) can be used to study problems of local controllability and to construct asymptotically stabilizing feedback controls for affine nonlinear control systems. Some interesting results in this sense have been recently obtained by $\mathrm{H}$. Hermes in [14] where it is shown that non-resonance conditions (as stated in Corollary 3.9 here) and small time local controllability (STLC) imply many standard 
necessary conditions for the existence of a continuous, asymptotically stabilizing feedback control (ASFC), for an $n$-dimensional, single input affine control system.

\section{ACKNOWLEDGEMENTS}

The content of this paper constitutes part of the author's Ph.D. dissertation at the University of Colorado at Boulder. The author would like to express his sincere thanks to Prof. Henry Hermes for his guidance, help and support. He also would like to thank Prof. Alberto Bressan for various fruitful discussions and comments on this work. Finally he thanks NSF for partially supporting this research through grant \# DMS 9301039.

\section{REFERENCES}

[1] A. A. Agrachev, R. V. Gamkrelidze and A. V. SarycheV, Local invariants of smooth control systems, Acta Applicandae Mathematicae, Vol. 14, 1989, pp. 191-237.

[2] F. ANCONA, Homogeneous normal forms for vector fields with respect to an arbitrary dilation, Ph. D. Dissertation, University of Colorado, Boulder, December 1993.

[3] V. I. ARNOLD, Geometrical Methods in the Theory of Ordinary Differential Equations, Springer-Verlag, New York, Heidelberg, Berlin, 1982.

[4] R. M. BIANCHINI and G. STEFANI, Graded approximations and controllability along a trajectory, SIAM J. Control Optim., Vol. 28, 1990, pp. 903-924.

[5] A. BRESSAN, Local asymptotic approximations of nonlinear control systems, Internat. $J$. Control., Vol. 41, 1985, pp. 1331-1336.

[6] K. T. CHEN, Equivalence and decomposition of vector fields about an elementary critical point, Amer. J. Math., Vol. 85, 1963, pp. 693-722.

[7] C. El.phick, E. Tirapegui, M. E. Brachet, P. Coullet and G. Iooss, A simple global characterization for normal forms of singular vector fields, Physica, Vol. 29D, 1987, pp. 85-127.

[8] J. Guckenheimer and P. Holmes, Nonlinear Oscillations, Dynamical Systems, and Bifurcations of Vector Fields, Appl. Math. Sci., Springer-Verlag, New York, Heidelberg, Berlin, 1983.

[9] H. HERMES, Nilpotent approximations of control systems and distributions, SIAM J. Control Optim., Vol. 24, 1986, pp. 731-736.

[10] H. HERMES, Homogeneous coordinates and continuous asymptotically stabilizing feedback controls, in: Differential Equations, Stability and Control (S. Elaydi, Ed.), Lecture Notes in Pure and Applied Mathematics, Vol. 127, pp. 249-260, Dekker, New York, 1991.

[11] H. HERMES, Asymptotically stabilizing feedback controls and the nonlinear regulator problem, SIAM J. Control Optim., Vol. 29, 1991, pp. 185-196.

[12] H. HeRmES, Nilpotent and higher order approximations of vector field systems, SIAM Review, Vol. 33, 1991, pp. 238-264.

[13] H. Hermes, Asymptotically stabilizing feedback controls, J. Diff. Eqns., Vol. 92, 1991, pp. 76-89.

[14] H. HERMES, Resonance and continuous asymptotically stabilizing feedback controls, Proc. IFAC NOLCOS 95 (to appear). 
[15] J. E. HumpHREYS, Introduction to Lie algebras and Representation Theory, Springer-Verlag, New York, Heidelberg, Berlin, 1972.

[16] M. KaWSKI, Stabilization of nonlinear systems in the plane, System Control Lett., Vol. 12, 1989, pp. 169-175.

[17] P. J. OLvER, Applications of Lie groups to Differential Equations, Springer-Verlag, New York, Heidelberg, Berlin, 1993.

[18] L. P. Rothschild and E. M. STEIN, Hypoelliptic differential operators and nilpotent groups, Acta Math., Vol. 137, 1976, pp. 247-320.

[19] G. STEFAni, Polynomial approximations to control systems and local controllability, Proc. 24th IEEE Conference on Decision and Control, Vol. I, 1985, pp. 33-38.

[20] H. J. Sussmann, A general theorem on local controllability, SIAM J. Control Optim., Vol, 25, 1987, pp. 158-194.

[21] A. VANDERBAuWHEDE, Center manifolds, normal forms and elementary bifurcations, in Dynamics Reported, U. Kirchgraber and H. O. Walther Ed., Vol. 2, pp. 89-169, Teubner, Stuttgart and Wiley, Chichester, 1989.

[22] J. C. VAN DER MEER, Nonsemisimple $1: 1$ resonance at an equilibrium, Cel. Mech., Vol, 27, 1982, pp. 131-149.

[23] J. C. VAN DER MEER, The Hamiltonian-Hopf Bifurcation, Lecture Notes in Math., Vol. 1160, Springer-Verlag, New York, Heidelberg, Berlin, 1985.

[24] V. S. VARAdARAJAN, Lie Groups, Lie Algebras and Their Representations, Springer-Verlag, New York, Heidelberg, Berlin, 1984.

(Manuscript received April 25, 1994; revised version received February 8, 1995.) 